\title{
Accuracy of Electrocardiographic Imaging using the Method of Fundamental Solutions
}

\author{
Peter R. Johnston ${ }^{\mathrm{a}, *}$ \\ ${ }^{a}$ School of Environment and Science and Queensland Micro- and Nanotechnology Centre, Griffith University, \\ Nathan, Queensland, Australia, 4111
}

\begin{abstract}
Solving the inverse problem of electrocardiology via the Method of Fundamental Solutions has been proposed previously. The advantage of this approach is that it is a meshless method, so it is far easier to implement numerically than many other approaches. However, determining the heart surface potential distribution is still an ill-posed problem and thus requires some form of Tikhonov regularisation to obtain the required distributions.

In this study, several methods for determining an "optimal" regularisation parameter are compared in the context of solving the inverse problem of electrocardiology via the Method of Fundamental Solutions. It is found that the Robust Generalised Cross-Validation method most often yields epicardial potential distributions with the least relative error when compared to the input distribution. The study also compares the inverse solutions obtained with the Method of Fundamental Solutions with those obtained in a previous study using the boundary element method. It is found that choosing the best solution methodology and regularisation parameter determination method depends on the particular scenario being considered.

Keywords: Inverse Problems; Electrocardiology; Tikhonov Regularisation; Method of Fundamental Solutions; Electrophysiology.
\end{abstract}

*Corresponding Author 


\section{Introduction}

One possible question to ask when solving the inverse problem of electrocardiology is: What is the heart surface potential distribution that gives rise to the observed body surface potential distribution? Generally, solving such a problem is a two step procedure.

The first step is to solve for the electric potential $\phi$, within the human thorax $\Omega$, in order to relate the heart surface potential distribution to the body surface potential distribution. The second step, solving the inverse problem, is to determine the heart surface potential distribution given a set of measured body surface potentials (generally recorded using a mapping system at a set of defined points on the torso surface).

Generally, the matrix that relates the heart surface potentials to the body surface potentials is not square and has a large condition number. Further, since there is often measurement noise in the body surface potential measurements, the inverse problem is ill-posed in the sense of Hadamard [1]. To overcome this ill-posedness, Tikhonov regularisation is used [34], but the drawback here is that a new, unknown, parameter (called the regularisation parameter) is introduced, thus making it difficult to find the heart surface potential distribution. Over many years, a number of methods have been proposed to find an "optimal" regularisation parameter for the inverse problem. Some examples include: the L-Curve method [2], the CRESO method [3], the Zero-Crossing method [4], the Generalised Cross-Validation method (GCV) [5], and the Robust Generalised Cross-Validation method (RGCV) [6]. A recent paper compared the performance of these methods in the context of the boundary element method solution of the forward problem and found that the RGCV would consistently outperform the other methods [7]. There have been several other methods for determining $\lambda$ in a more general context and these are reviewed in [8].

The methods, described above, all use Tikhonov regularisation to constrain the spatial variation of the epicardial potential distribution. There are also methods that take into account the temporal variation $[9,10,11,12]$, but these also require some form of optimal regularisation [13].

There are also alternative methods for solving the inverse problem of electrocardiology that do not rely on regularisation techniques. These include using: genetic algorithms $[14,15,16]$; partial differential equation constrained optimisation $[17,18]$; Twomey regularisation for wave- 
front based ECG imaging [19], and Bayesian estimation [20]. Some more recent methods for solving the inverse problem of electrocardiology include a Steklov-Poincaré variational formulation, [21], the factorisation method of boundary value problems [22], and methods that use electrical and mechanical measurements [23].

An alternative approach for solving the inverse problem of electrocardiology was proposed several years ago [24]. The underlying numerical method, used to solve the forward problem in that case, was the Method of Fundamental Solutions (MFS) [25]. This approach also yields a matrix-vector equation as the solution of the equation governing the electric field, and Tikhonov regularisation is still required to obtain an inverse solution. The key difference between the MFS approach and the more usual volume conductor approaches is that the MFS inverse problem seeks to find the coefficients in the expansion of the solution, instead of the potentials directly. For the MFS approach, the final epicardial potential distribution is found as a postprocessing step once the coefficients have been obtained. One advantage of the MFS approach is that it is a "meshless" method, meaning that the solution can be found simply by knowing the positions of the nodes in the bounding surfaces, without the requirement for a mesh linking the nodes together.

The MFS approach has been applied to various inverse problems previously [26, 27] and, as mentioned above, in particular to the inverse problem of electrocardiology [24]. Recent applications of the MFS to ECGI include studies of the locations of the heart and torso boundaries [28], application of the U-curve and the discrete Picard condition [29, 30].

The purpose of this paper is to compare the performance of the various regularisation parameter determination methods when they are applied to the MFS approach. Solutions found via this approach will be compared to a previous study [7], which uses the Boundary Element Method (BEM) as a method for solving the forward problem. Both the BEM and MFS methods assume that the thorax is homogeneous; however, this may easily be relaxed with the BEM, but not so easily relaxed with the MFS.

The remainder of the paper is structured as follows. The next section introduces the MFS in the context of the inverse problem of electrocardiology and discusses the implementation of the inverse problem. Further, the regularisation parameter determination methods to be considered here are also briefly introduced in this section. Section 3 presents the results of the comparisons 
between the different methods of choosing the regularisation parameter, and Section 4 discusses the merits of each method and compares the performance of the methods, in the context of MFS, with the previously published performance of the methods, in the context of the BEM [7]. Finally, Section 5 makes some recommendations regarding the various approaches.

\section{Methods}

\subsection{Governing Equations}

Assume that the electric potential within the human thorax, $\Omega$, is governed by Laplace's equation [31]

$$
\nabla \cdot \sigma \nabla \phi=0 \quad \text { in } \quad \Omega
$$

subject to a known potential distribution, $h(\mathbf{x})$, on the heart surface, $\Gamma_{h}$

$$
\phi=h(x, y, z) \quad \text { on } \quad \Gamma_{h}
$$

and assuming that the body surface, $\Gamma_{b}$, is insulated,

$$
(\sigma \nabla \phi) \cdot \mathbf{n}=0 \quad \text { on } \quad \Gamma_{b}
$$

where $\sigma$ is the conductivity tensor within the torso and $\mathbf{n}$ is the outward pointing normal from the body surface.

After approximating the thorax by a mesh, the governing equation (1) can be solved by one of several numerical methods, (for example, the finite difference method, the finite element method, the finite volume method and the boundary element method [32]) to relate the body surface potentials, $\mathbf{b}$, to the heart surface potentials, $\mathbf{h}$, in a matrix equation

$$
\mathrm{Ah}=\mathbf{b}
$$

where $\mathbf{A}$ is known as the forward transfer matrix [33]. An excellent review of these volume conductor approaches to solving the forward problem can be found in [1]. An alternative approach, which yields a similar formulation is given in terms of current dipoles, and this is also reviewed in [1]. 


\subsection{The Inverse Problem}

As mentioned above, since the coefficient matrix $\mathbf{A}$ is not square and has a large condition, the inverse problem is ill-posed. Therefore, solving equation (4) requires Tikhonov regularisation [34], which gives $\mathbf{h}$ by finding

$$
\min _{\mathbf{h}}\left\{\|\mathbf{A h}-\mathbf{b}\|_{2}^{2}+\lambda^{2}\|\mathbf{L h}\|_{2}^{2}\right\}
$$

The $\mathbf{L}$ matrix can be an appropriately sized identity matrix, or an approximation to the gradient or Laplacian operators on the surface of the heart, yielding zero-, first- or second-order Tikhonov regularisation, respectively. Minimisation of equation (5) gives

$$
\mathbf{h}=\left(\mathbf{A}^{T} \mathbf{A}+\lambda^{2} \mathbf{L}^{T} \mathbf{L}\right)^{-1} \mathbf{A}^{T} \mathbf{b}
$$

where $\lambda$ is the regularisation parameter. Since the $\mathbf{L}$ matrix affects the smoothing of the epicardial potential distribution, the parameter $\lambda$ performs the task of balancing the amount of smoothing (second term in equation (5)) with the size of the residual (first term in equation $(5))$.

\subsection{The Method of Fundamental Solutions}

The Method of Fundamental Solutions (MFS) was introduced in the late 1970s as a method to solve boundary value problems similar to those given by equations (1), (2) and (3). Of interest here is the fact that this approach has been used to solve the inverse problem of electrocardiology [24]. This formulation solves Laplace's equation (1) along with the insulation condition (3), and the extra condition

$$
\phi(\mathbf{x})=m(\mathbf{x}), \quad \mathbf{x} \in \Gamma_{J} \subset \Gamma_{b}
$$

where $\Gamma_{J}$ represents a set of points, of size $N$, say, at which the electric potential has been measured on the torso surface, $\Gamma_{b}$. This subset of the torso potentials can be thought of as body surface mapping jacket. In this formulation, both the body surface potentials and their normal gradient are specified; that is, both Dirichlet and Neumann boundary conditions are imposed on the one surface and this is referred to as a "Cauchy" problem. As mentioned previously, determining the heart surface potentials, or solving the inverse problem, is a postprocessing step. 
Like the BEM, MFS is also a boundary method and it utilises the same fundamental solution

$$
f(r)=\frac{1}{4 \pi r}
$$

where $r=\|\mathbf{x}-\mathbf{y}\|$ represents the distance between some observation point $\mathbf{x}$ and some source point $\mathbf{y}$. However, unlike the BEM, singular integrals are avoided by choosing a set of source points $\left\{\mathbf{y}_{j}\right\}$ lying outside the computational domain. Since the computational domain for this problem is the space between the heart surface and the body surface, source points are created by pushing the torso mesh points outside the torso and shrinking the heart mesh points inside the heart.

To solve Laplace's equation (1) via MFS, assume a solution of the form

$$
\phi(\mathbf{x})=c_{0}+\frac{1}{4 \pi} \sum_{j=1}^{M} \frac{c_{j}}{\left\|\mathbf{x}-\mathbf{y}_{j}\right\|}
$$

with $M=M_{T}+M_{H}$, where $M_{T}$ and $M_{H}$ are the number of nodes in the torso and heart meshes, respectively. It now follows that

$$
\frac{\partial \phi}{\partial n}=\sum_{j=1}^{M} \frac{\partial}{\partial n}\left(\frac{c_{j}}{4 \pi\left\|\mathbf{x}-\mathbf{y}_{j}\right\|}\right)
$$

Now, assuming that the electric potential can be measured at the $N$ positions $\mathbf{x}_{i}, i=$ $1, \ldots, N$ (via a mapping jacket system), then

$$
c_{0}+\sum_{j=1}^{M} \frac{c_{j}}{4 \pi\left\|\mathbf{x}_{i}-\mathbf{y}_{j}\right\|}=m\left(\mathbf{x}_{i}\right), \quad i=1, \ldots, N,
$$

where $m\left(\mathbf{x}_{i}\right)$ represents the sampled electric field. Also, at these electrode positions, it is assumed that the body surface is insulated, and so it follows that

$$
\frac{1}{4 \pi} \sum_{j=1}^{M} \frac{\partial}{\partial n}\left(\frac{c_{j}}{\left\|\mathbf{x}_{i}-\mathbf{y}_{j}\right\|}\right)=0, \quad i=1, \ldots, N .
$$

Combining these two equations yields a $2 N \times(M+1)$ system of algebraic equations

$$
\mathrm{Ac}=\mathbf{b}
$$


in the coefficients $c_{j}, j=1, \ldots, M$. In this system of equations

$$
\mathbf{A}=\left(\begin{array}{cccc}
1 & \frac{1}{4 \pi\left\|\mathbf{x}_{1}-\mathbf{y}_{1}\right\|} & \cdots & \frac{1}{4 \pi\left\|\mathbf{x}_{1}-\mathbf{y}_{M}\right\|} \\
\vdots & \vdots & \cdots & \vdots \\
1 & \frac{1}{4 \pi\left\|\mathbf{x}_{N}-\mathbf{y}_{1}\right\|} & \cdots & \frac{1}{4 \pi\left\|\mathbf{x}_{N}-\mathbf{y}_{M}\right\|} \\
0 & \frac{\partial}{\partial n}\left(\frac{1}{4 \pi\left\|\mathbf{x}_{1}-\mathbf{y}_{1}\right\|}\right) & \cdots & \frac{\partial}{\partial n}\left(\frac{1}{4 \pi\left\|\mathbf{x}_{1}-\mathbf{y}_{M}\right\|}\right) \\
\vdots & \vdots & \cdots & \vdots \\
0 & \frac{\partial}{\partial n}\left(\frac{1}{4 \pi\left\|\mathbf{x}_{N}-\mathbf{y}_{1}\right\|}\right) & \cdots & \frac{\partial}{\partial n}\left(\frac{1}{4 \pi\left\|\mathbf{x}_{N}-\mathbf{y}_{M}\right\|}\right)
\end{array}\right)
$$

$\mathbf{c}=\left(c_{0}, c_{1}, \ldots, c_{M}\right)^{T}$ and $\mathbf{b}=\left(m\left(\mathbf{x}_{1}\right), m\left(\mathbf{x}_{2}\right), \ldots, m\left(\mathbf{x}_{N}\right), 0, \ldots, 0\right)^{T}$.

Note that, generally, $2 N<M+1$, so the system of equations (13) in underdetermined. Despite the fact that this system is underdetermined, Tikhonov regularisation can still be used to find the coefficients $c_{j}, j=1, \ldots, M$, in equation (13) utilising the methods for regularisation parameter determination discussed in [7]. Once these coefficients have been determined, the heart surface potential distribution can be calculated via

$$
\phi_{H}(\mathbf{x})=c_{0}+\frac{1}{4 \pi} \sum_{j=1}^{M} \frac{c_{j}}{\left\|\mathbf{x}-\mathbf{y}_{j}\right\|} \quad \text { for } \quad \mathbf{x} \in \Gamma_{H}
$$

\subsection{Solving the Inverse Problem}

\subsubsection{Singular Value Decomposition}

Singular value decomposition (SVD) is a useful tool to choose "optimal" regularisation parameters in the inverse problem of electrocardiology. For an $m \times n$ matrix $\mathbf{A}$, which can be overdetermined $(m>n)$ from the BEM or underdetermined $(m<n)$ from MFS, A can be written as

$$
\mathbf{A}=\mathbf{U} \mathbf{\Sigma} \mathbf{V}^{T}
$$

where $\mathbf{U}$ is an $m \times m$ orthogonal matrix, $\mathbf{V}$ is an $n \times n$ orthogonal matrix and $\boldsymbol{\Sigma}$ is an $m \times n$ diagonal matrix with the singular values of $\mathbf{A}$ as the diagonal elements and ordered such that $\sigma_{1} \geq \sigma_{2} \geq \ldots \sigma_{\min (m, n)} \geq 0$ [35]. Assuming that $p=\min (m, n)$, equation (16) can be rewritten as

$$
\mathbf{A}=\sum_{i=1}^{p} \mathbf{u}_{i} \sigma_{i} \mathbf{v}_{i}^{T}
$$

where $\mathbf{u}_{i}, i=1, \ldots, p$ are the first $p$ columns of $\mathbf{U}$ and $\mathbf{v}_{i}, i=1, \ldots, p$ are the first $p$ columns of $\mathbf{V}[35]$. 


\subsubsection{Obtaining the Inverse Solution}

For the purposes of this study, only zero-order Tikhonov regularisation will be considered. There are two reasons for this choice. Firstly, it will allow direct comparison with a previous study of this nature [7]. Secondly, higher order Tikhonov regularisation asserts some form of smoothing on the solution of the inverse problem, which is appropriate when solving directly for the electric potentials (for example, using a BEM approach); however, for MFS, a series of coefficients is sought and there is nothing to suggest that their distribution needs to be smoothed.

Hence, the object is to find

$$
\min _{\mathbf{h}}\left\{\|\mathbf{A h}-\mathbf{b}\|_{2}^{2}+\lambda^{2}\|\mathbf{h}\|_{2}^{2}\right\}
$$

the solution of which is [36]

$$
\mathbf{h}=\left(\mathbf{A}^{T} \mathbf{A}+\lambda^{2} \mathbf{I}\right)^{-1} \mathbf{A}^{T} \mathbf{b}=\sum_{i=1}^{p} \frac{\sigma_{i}^{2}}{\sigma_{i}^{2}+\lambda^{2}} \frac{\alpha_{i}}{\sigma_{i}} \mathbf{v}_{i}
$$

where $\alpha_{i}=\mathbf{u}_{i}^{T} \mathbf{b}$. Further, it can also be shown that the two terms in the zero-order Tikhonov functional (18) can be written as [4],

$$
\rho^{2}(\lambda):=\|\mathbf{A h}-\mathbf{b}\|_{2}^{2}=\sum_{i=1}^{p} \frac{\lambda^{2} \alpha_{i}^{2}}{\left(\sigma_{i}^{2}+\lambda^{2}\right)^{2}}+\left\|\mathbf{r}_{\perp}\right\|_{2}^{2}
$$

and

$$
\eta^{2}(\lambda):=\|\mathbf{h}\|_{2}^{2}=\sum_{i=1}^{p} \frac{\sigma_{i}^{2} \alpha_{i}^{2}}{\left(\sigma_{i}^{2}+\lambda^{2}\right)^{2}}
$$

where $\left\|\mathbf{r}_{\perp}\right\|_{2}^{2}=\left\|\mathbf{A} \mathbf{x}_{L S S}-\mathbf{b}\right\|_{2}^{2}$ is the residual from the least squares solution to equation (4).

\subsection{Choosing the Regularisation Parameter}

This study will focus on the following methods for approximating the "optimal" regularisation parameter. These methods have been introduced more fully in [7] and will only be discussed briefly here.

\subsubsection{The L-Curve Method}

A plot on a log-log scale of $\rho(\lambda)$ vs $\eta(\lambda)$ is called the L-Curve [2]. It is generally accepted that an approximation to the optimal value of $\lambda$ occurs at the corner of the L-Curve where the corner is defined as one of the two possibilities: 
1. the point that is closest to the origin, or

2. the point of maximum curvature along the curve.

In this study the latter approach will be used.

\subsubsection{The CRESO Method}

The Composite REsidual and Smoothing Operator (CRESO) method [3], which has already been applied to the MFS solution to the inverse problem of electrocardiology [24], predicts an

estimate for the optimal regularisation parameter that corresponds to the first local maximum of the function

$$
C(\lambda)=\frac{d}{d\left(\lambda^{2}\right)}\left(\lambda^{2} \eta^{2}(\lambda)-\rho^{2}(\lambda)\right)
$$

where the derivative is with respect to $\lambda^{2}$. In terms of the singular value decomposition, this can be rewritten as [4]

$$
C(\lambda)=\sum_{i=1}^{p} \frac{\sigma_{i}^{2} \alpha_{i}^{2}\left(\sigma_{i}^{2}-3 \lambda^{2}\right)}{\left(\sigma_{i}^{2}+\lambda^{2}\right)^{3}} .
$$

\subsubsection{The Zero-Crossing Method}

The Zero-Crossing method [4] seeks to find a balance between the solution norm and the residual norm by estimating the optimal regularisation parameter as a zero of the function

$$
B(\lambda)=\lambda^{2} \eta^{2}(\lambda)-\rho^{2}(\lambda)
$$

which, in terms of the SVD, can be rewritten as

$$
B(\lambda)=\sum_{i=1}^{p} \frac{\lambda^{2} \alpha_{i}^{2}\left(\sigma_{i}^{2}-\lambda^{2}\right)}{\left(\sigma_{i}^{2}+\lambda^{2}\right)^{2}}-\left\|\mathbf{r}_{\perp}\right\|_{2}^{2}
$$

\subsubsection{The Generalised Cross-Validation Method}

The Generalised Cross-Validation (GCV) [5] method provides an estimate of the optimal regularisation parameter by seeking to minimise the function

$$
G(\lambda)=\frac{\rho^{2}(\lambda)}{\left[\operatorname{Tr}\left(\mathbf{I}-\mathbf{A} \mathbf{A}^{\#}\right)\right]^{2}}
$$

where $\mathbf{A}^{\#}=\left(\mathbf{A}^{T} \mathbf{A}+\lambda^{2} \mathbf{I}\right)^{-1} \mathbf{A}^{T}$ is the regularised inverse of $\mathbf{A}$ and $\operatorname{Tr}(\cdot)$ is the trace operator of a matrix. In terms of the SVD, $G(\lambda)$ can be rewritten

$$
G(\lambda)=\frac{\sum_{i=1}^{p} \frac{\lambda^{4} \alpha_{i}^{2}}{\left(\lambda^{2}+\sigma_{i}^{2}\right)^{2}}+\left\|\mathbf{r}_{\perp}\right\|_{2}^{2}}{\left(m-\sum_{i=1}^{p} \frac{\sigma_{i}^{2}}{\lambda^{2}+\sigma_{i}^{2}}\right)^{2}}
$$




\subsubsection{The Robust Generalised Cross-Validation Method}

The Robust Generalised Cross-Validation (RGCV) method was introduced in [6] and estimates the optimal regularisation parameter $\lambda$ as the value of $\lambda$ that minimises the $\mathrm{RGCV}$ function

$$
\bar{G}(\lambda)=\{\gamma+(1-\gamma) \mu(\lambda)\} G(\lambda)
$$

where $G(\lambda)$ is given by equation $(26)$ and $\mu(\lambda)$ is defined as

$$
\mu(\lambda)=\left[\operatorname{Tr}\left(\mathbf{A A}^{\#}\right)\right]^{2}=\sum_{i=1}^{p} \frac{\sigma_{i}^{4}}{\left(\lambda^{2}+\sigma_{i}^{2}\right)^{2}}
$$

using the SVD.

Equation (28) also contains the so-called robustness parameter, $\gamma$, where $\gamma \in[0,1]$. A previous study [7] has demonstrated that for ECGI it is practical to fix $\gamma=0$ and so this will also be assumed here.

\subsection{Simulation Protocols}

The simulation protocols used to compare the above methods for determining an approximation to the optimal regularisation parameter are essentially the same as those detailed

previously [4, 7]. A simple heart-torso system similar to many tank experiments [37], forms the basis of the geometrical model considered. The torso model [38] was $550 \mathrm{~mm}$ high, with a width of $326 \mathrm{~mm}$ under the arms and $223 \mathrm{~mm}$ from front to back and was created with 610 nodes and 1220 triangles, as shown in Figure 1. Two heart meshes [39], consisting of the outer surface of the ventricles and atria, both $132 \mathrm{~mm}$ from base to apex and approximately $90 \mathrm{~mm}$ in diameter, were also used, one containing 610 nodes and the other with 114 nodes. The 114 node mesh was chosen to reflect typical epicardial sock electrode numbers and was subsampled from the 610 node mesh. This represents an attempt to determine realistically a discrete sample from what is essentially a continuous distribution.

Epicardial potential distributions for a normal heartbeat and an ectopic beat were generated from a cellular automata heart model [39]. These distributions were projected onto the two heart meshes at 1 millisecond time intervals.

The boundary element method, with the heart in the "correct" position, was used to create a $610 \times 610$ forward transfer matrix, which was used to create body surface potential maps at each 
time instant. Measurements from jacket, as shown in Figure 2, consisting of 168 electrodes, was subsampled from the 610 node body surface potential distributions. This high resolution heart mesh was used to create "true" body surface potential maps. The MFS method was applied to heart-torso geometries with the heart in the correct position, as well as with introduced geometry noise created by moving the heart $\pm 10 \mathrm{~mm}$ in each of the three coordinate directions.

"White" measurement noise was also added to the body surface potential distributions using the root mean square values of the heart surface potential at noise levels of $1 \%, 2 \%, 5 \%$, $10 \%$ and $20 \%$. Each resulting body surface potential distribution was then used with MFS to determine a 114 node epicardial potential distribution, which was in turn compared with the 114 node epicardial potential distribution as sampled from the original 610 node distribution.

The MFS method was implemented by moving the torso nodes $40 \mathrm{~mm}$ outward, in the normal direction, from the original surface and the heart nodes $10 \mathrm{~mm}$ inward, in the normal direction (Figure 3). These values were found to give the most consistent accuracy of the inverse solution across the simulations performed and regularisation parameter determination methods considered. However, these values may not be appropriate for a different heart-torso combination.

Two heartbeat scenarios were also considered: a normal activation sequence over the QRS complex (175ms to $245 \mathrm{~ms}$ after onset of the beat), and an ectopic beat, triggered in the lateral left ventricle of the heart model (90ms to $160 \mathrm{~ms}$ after pacing). Both simulations were studied at $10 \mathrm{~ms}$ intervals.

\subsection{Measures of Accuracy}

Since this is a simulation study and the desired epicardial potential distribution is known, it is possible to gauge the accuracy of the inverse solution by either the relative error $(R E)$, namely,

$$
R E(\lambda)=\frac{\left\|\mathbf{x}_{\lambda}-\mathbf{x}_{E}\right\|}{\left\|\mathbf{x}_{E}\right\|}
$$

or the correlation coefficient $(C C)$, given by

$$
C C(\lambda)=\frac{\sum_{i=1}^{n}\left[\left(\mathbf{x}_{\lambda}\right)_{i}-\overline{\mathbf{x}_{\lambda}}\right]\left[\left(\mathbf{x}_{E}\right)_{i}-\overline{\mathbf{x}_{E}}\right]}{\left\|\mathbf{x}_{\lambda}-\overline{\mathbf{x}_{\lambda}}\right\|\left\|\mathbf{x}_{E}-\overline{\mathbf{x}_{E}}\right\|} .
$$

In Equations (30) and (31), $\mathbf{x}_{E}$ is the known epicardial potential distribution, and $\mathbf{x}_{\lambda}$ the computed one. The quantities $\overline{\mathbf{x}_{E}}$ and $\overline{\mathbf{x}_{\lambda}}$ are, respectively, the mean values of $\mathbf{x}_{E}$ and $\mathbf{x}_{\lambda}$ over 
the $n$ epicardial sites.

For the remainder of the paper, a solution will be referred to as "optimal" if it is the solution that yields the minimum relative error over all values of $\lambda$.

\section{Results}

\subsection{Comparison of the Inversion Techniques}

Tables 1 and 2 show how often each regularisation parameter determination method yields the lowest relative error for the normal and ectopic beats, respectively, at different levels of added noise. The tables indicate that the RGCV method gives the lowest relative error at most noise levels across both types of heart beat. The one exception is for the ectopic beat with $10 \%$ added noise (Table 2), where the GCV method most often yields the least relative error. The tables also show that either the GCV or CRESO method is most often the second best performing method, with the GCV method generally yielding a lower relative error at higher noise levels. The L-Curve and Zero-Crossing methods generally perform quite poorly by comparison with the other methods.

Tables 3 and 4 demonstrate how the regularisation parameter determination methods compare over the time course of the normal and ectopic beats, respectively. Across both types of beat, the RGCV method most often yields the least relative error except for one time instant in both beats. At $215 \mathrm{~ms}$ in the normal beat (Table 3) and $140 \mathrm{~ms}$ in the ectopic beat (Table 4), the L-Curve method most often yields the smallest relative error, followed closely by the CRESO method. Also, at 90ms in the ectopic beat (Table 4), the GCV method yields the smallest relative error. Generally, the CRESO method is the second best performing method across the normal heartbeats and the GCV method is the second best across the ectopic beats.

Finally, Tables 5 and 6 compare how the different regularisation parameter determination methods perform with various heart position offsets for the normal and ectopic heart beats, respectively. For the normal beat (Table 5), RGCV most often yields the least relative error at all offset values. For the ectopic beat (Table 6), RGCV most often gives the least relative error at four offset positions and the GCV method is better for the remaining positions. The CRESO method is generally the next best performed method. 
In summary, for the 280 different simulations performed, the RGCV method most often produces an epicardial potential distribution with the least relative error on 148 occasions for the normal heart beat and 131 occasions for the ectopic beat. For the normal beat the next best performing method is the CRESO method, producing the least relative error on 50 occasions. However, for the ectopic beat, the GCV method is the second best performing method producing the least relative error on 90 occasions.

\subsection{Reconstructed Epicardial Potential Distributions}

Another aspect of this study is to compare solutions of the inverse problem of electrocardiology obtained from the MFS approach to previously published solutions obtained from a BEM solution of the forward problem [7]. With this is mind, the following results are presented to allow a direct comparison of the epicardial potential distributions. This is achieved by presenting results at the same time points of the normal and ectopic beats and at the same noise levels and heart offsets as those previously published [7].

Figures 4 and 5 show the input epicardial potential distributions for the normal and ectopic beats, respectively, at the indicated time points. The left hand column of both figures shows the original 610 node epicardial potential distribution used to generate the body surface potential maps and the right hand column is the subsampled 114 node epicardial potential distribution used to compare the calculated inverse solutions. See [7] for a complete discussion of the motivation behind this methodology.

Figure 6 presents inverse epicardial potential distributions obtained from the indicated regularisation parameter determination methods at $205 \mathrm{~ms}$ after the start of the normal heartbeat with the heart offset to the left by $10 \mathrm{~mm}$ and with the addition of $1 \%$ RMS noise to the body surface potential map. The optimal regularisation parameter is also shown in each case. It can be seen from the figures that the RGCV method yields an epicardial potential distribution that is very close to the optimal solution in the sense of having the least relative error and highest correlation coefficient. The GCV method is a close second based on these measures. Both the CRESO and L-Curve approaches produce potential distributions that are over-regularised. Also from the figure it can be seen that the RGCV and GCV solutions have the minimum in the same place as the optimal solution and this is a good approximation to the position of the minimum in the target epicardial potential distributions (Figures 4(a) and 4(b)). However, it 
is interesting to observe that, while both RGCV and GCV solutions have their maxima at the same position (which is different from that in the optimal solution), these maxima are very close to the positions of the maxima of the input epicardial potential distributions.

Another point of comparison between the inverse solutions and the input epicardial potential distributions is the range of potentials obtained. For the optimal, RGCV and GCV solutions, the range of potentials is smaller and is shifted in the negative direction. The GCV solution has the range of potentials that is closest to the input range, followed by the RGCV solution. The remaining regularisation parameter determination methods all yield solutions that are massively over-regularised, smearing out most of the desired detail.

Figure 7 shows the reconstructed epicardial potential distributions from the indicated regularisation parameter determination methods, with the specified regularisation parameters, at $235 \mathrm{~ms}$ into the normal heart beat with the heart offset $10 \mathrm{~mm}$ in the positive $z$ direction and 20\% added RMS noise. Here the RGCV, GCV and CRESO methods all yield inverse solutions that are close to optimal, with all three placing the maxima and minima in the same positions as the optimal. Note that the RGCV method yields a higher correlation coefficient than the optimal solution, as does the GCV method. In this case, only the minima of the inverse epicardial potential distributions is close to that of the original distribution (Figure 4(d)), with the maximum being placed on the opposite side of the heart. Again, the range of the inverse potentials is smaller than the input range. This is a more testing case with $20 \%$ noise added to the body surface potential map and the heart moved deeper inside the torso, and this is reflected in the higher relative error and lower correlation coefficient. Both the L-Curve and the Zero-Crossing methods produce solutions that are over-regularised and hence far too smooth, showing very little variation in potential across the heart surface.

Figure 8 shows inverse epicardial potential distributions obtained for the indicated regularisation parameter determination methods, with the indicated regularisation parameters, 130ms into the ectopic beat, with the heart moved $10 \mathrm{~mm}$ in the negative $z$ direction (closer to the torso surface) and with the addition of $10 \%$ RMS noise. Again, the RGCV, GCV and CRESO solutions all look very similar and all have their maxima and minima in the same places as the optimal solution. However, the relative error for RGCV is the lowest of all the solutions, with the highest correlation coefficient. As in the previous example, the minimum of each inverse 
distribution is close to that of the input distribution (Figure 5(b)). Also, here, the position of the maxima are close to that of the input distribution. Again, the Zero-Crossing and the L-Curve solutions are over-regularised.

The final example (Figure 9) shows the inverse epicardial potential distributions obtained at $160 \mathrm{~ms}$ into the ectopic beat with $1 \%$ added RMS noise and the heart moved $10 \mathrm{~mm}$ in the positive $x$ direction. Here, only the RGCV and GCV solutions are close to the optimal solution. They all have maxima and minima in the same positions and these are reasonably close to those of the original distributions. Also, the RMS values for the resulting epicardial potential distributions are much closer to those of the original distribution than any of the previous examples considered. In this case, the CRESO, L-Curve and Zero-Crossing methods all perform poorly.

As part of an overall summary of the performance of the regularisation parameter determination methods, Figure 10 gives a summary of the means and standard deviations of the relative errors for 20 realisations across the QRS complex of the normal heart beat with $5 \%$ RMS noise added to the body surface potential map and the heart offset $10 \mathrm{~mm}$ towards the back of the torso $(10 \mathrm{~mm}$ in the positive $z$ direction). It can be seen that, at most of the time points considered, the RGCV approach produces an inverse solution that closely matches the optimal inverse solution. This is followed closely by the solutions obtained from the GCV and CRESO approaches. The L-Curve and Zero-Crossing approaches generally result in the least accurate solutions. It is worth noting that the RGCV approach most often yields the smallest standard deviation over the realisations for this simulation scenario. Also of interest is the observation that during the latter half of the QRS complex the accuracy of all solutions obtained is similar and that the standard deviation of the values is also very small compared to those in the earlier part of the QRS complex.

Figure 11 shows a similar plot with $20 \%$ added noise and the heart offset $10 \mathrm{~mm}$ in the positive $z$ direction. In this case, the RGCV and GCV approaches admit solutions that are of similar accuracy and show similar magnitudes for the standard deviation across all time points in the QRS. For about half of the time points considered, the L-Curve and CRESO approaches yield solutions that are of similar accuracy and more accurate than the above two methods. The Zero-Crossing approach generally gives the least accurate solutions. For this scenario the 
CRESO and L-Curve approaches have the smallest standard deviations.

Figure 12 shows the relative errors across the QRS complex of the ectopic beat with $10 \%$ added RMS noise and the heart moved $10 \mathrm{~mm}$ towards the front of the torso (the negative $z$ direction). This shows that, at all time points considered, the RGCV and GCV methods produce solutions of similar accuracy with standard deviations of similar magnitudes. Further, again at all time points, these two approaches produce solutions that are closer to the optimal solution. Over most time points the CRESO approach yields the next most accurate solutions with the L-Curve and Zero-Crossing approaches being the next most accurate in about half of the time instances each. Interestingly, despite not providing the most accurate solutions at any time point, the L-Curve and Zero-Crossing methods have the smallest magnitudes over all the standard deviations.

Finally, Figure 13 shows the relative errors across the QRS complex of the ectopic beat with $1 \%$ added RMS noise and no offset in the heart position. Here it is a close tie between the RGCV and CRESO approaches to provide the most accurate solutions at most time points across the QRS complex. However, there are two instances when the RGCV is clearly superior to CRESO. It can also be seen from the figure that the standard deviation for the RGCV approach is always smaller than that of the CRESO method. At all time instants, the GCV approach results in the next best solutions. The L-Curve generally gives the next most accurate solutions, but there are two time instants where it gives solutions of similar accuracy to the RGCV approach. The Zero-Crossing approach performs worst at all time points.

\section{Discussion}

It is interesting to compare the accuracy and behaviour of the optimal regularisation parameter choosing methods, when applied to the boundary element method [7] and the method of fundamental solutions presented here.

Firstly, compare individual inverse solutions obtained at a single time instant with a given noise level and heart offset (Figures 6, 7, 8 and 9) here and in [7]. Comparing Figures 6 here and in [7], the RGCV method produces a more accurate inverse solution with MFS, but for all other methods the BEM yields the more accurate inverse solution. The case where the BEM solution yields a more accurate inverse solution across all methods is for the normal heartbeat, 
when there is $20 \%$ noise and the heart is offset deeper inside the torso (Figure 7); this is the most challenging example. In Figure 8 (for an ectopic beat) the MFS inverse solution gives a lower relative error and higher correlation coefficient than the BEM inverse solution for the CRESO, GCV and RGCV methods. Finally, for the ectopic beat shown in Figure 9, the MFS approach gives superior inverse solutions only for the RGCV and GCV methods.

Now, consider the overall performance across the QRS complex for both types of heartbeat (Figures 10, 11, 12 and 13). In Figures 10, at early time points in the QRS complex in the normal heart beat $(175 \mathrm{~ms}, 185 \mathrm{~ms})$, the BEM produces the more accurate solutions, whilst at $205 \mathrm{~ms}$ the MFS solutions are more accurate. For the remainder of the time points considered, the two methods yield solutions of similar accuracy. However, one observation is that, for the MFS approach, there is less variability between the parameter choosing approaches than with the BEM solutions and the standard deviations are much smaller. Similar comments apply to Figures 11, except that the BEM solutions are now more accurate for the entire first half of the QRS complex. For the ectopic beat, the MFS solutions are clearly better at 130ms and $140 \mathrm{~ms}$ of the QRS complex, worse at $90 \mathrm{~ms}$ and $150 \mathrm{~ms}$ and similar at the remaining time points (Figures 12). In this case, for the MFS approach, there is a greater variability in the accuracy of the various optimal regularisation parameter choosing methods than in the BEM approach. Finally, for Figures 13 the BEM approach results in solutions that are of similar or better accuracy than those obtained from the MFS approach at all time points across the QRS complex, except at $160 \mathrm{~ms}$.

Finally, a comparison of the number of times a regularisation parameter determination method gave the lowest relative error for the BEM and MFS approaches, over all 280 simulations for both beats, is given in Table 7. The table shows that for both the BEM and MFS, the RGCV method most often yields the least relative error across all simulations and for both heart beat types. The second best performing method varies with the situation. For the BEM, it is the L-Curve approach. However, for the MFS it is the CRESO method for a normal heart beat and the GCV for the ectopic beat.

The findings from this study generally agree with the findings of Wang and Rudy [24], that the MFS method can provide more accurate solutions than the BEM. However, the overall improvement suggested in [24] could not be reproduced here. It appears that the choice of MFS 
or BEM depends on the noise level in the measured body surface potentials, the position of the heart within the torso and the choice of method for determining the regularisation parameter. Only the CRESO method was used in [24].

The studies presented in $[29,30]$ also use the CRESO method as the gold standard and measure the improvements in their new methods by comparison with the CRESO solutions. It is difficult to draw a comparison between these studies and the one presented here as the $\mathrm{RE}$ and $\mathrm{CC}$ have different definitions. There is also no indication of any sources of noise in their study. From Figures 6 and 7, it can be seen that there is a reduction in RE (as defined here) of between $2.3 \%$ and $24.8 \%$ compared to the CRESO solution. This is a similar or better improvement than suggested in [29, 30]. Finally, Bouyssier et al. [22] present simulations over a heartbeat cycle with average $\mathrm{CC}$ of about 0.7 and average $\mathrm{RE}$ of about 0.85 , which is also in keeping with the data presented here.

One aspect of the MFS approach that was not considered in great detail as part of this study was the placement of the source points. Here the source points were moved outside of the computational domain in the normal direction to the inner and outer surfaces of the domain, as appropriate. A previous application of the MFS to ECGI [24] created the source points by moving the surface nodes along a ray, either inward or outward, as appropriate, joining the nodes to the centre of gravity of the heart. Other approaches to specifying the source nodes have also been presented elsewhere [28]. It is worth noting that it is possible to determine the number and position of the source nodes as part of the solution process, using non-linear optimisation routines [25]. The downside to this approach is that it would be computationally very expensive, especially in the context of the inverse problem of electrocardiology.

\section{Limitations}

One obvious limitation of this study is that only one heart-torso geometry has been considered. The next step for comparison would be to use several of the experimental data sets that are now in the public domain (for example the EDGAR data base available at www.edgar.sci.utah.edu). However, this study does provide a thorough treatment of noise sources and repeat runs that are not considered in other simulation studies. 


\section{Conclusion}

In conclusion, it appears from this study that the RGCV method (with $\gamma=0$ ) most consistently chose a regularisation parameter that gave the smallest relative error, when applying MFS to the inverse problem of electrocardiology. A similar conclusion was reached previously when using the BEM to solve the inverse problem. This is an interesting observation given that the Tikhonov functional that appears in both formulations seeks to obtain two different quantities: a potential distribution for the BEM and a series of coefficients in MFS, from which the potential distribution is calculated.

Combining the findings from this study with those given in [7] shows that there is no clearcut best choice for MFS over BEM. Different circumstances (for example, noise levels and heart offsets) call for different approaches to best solve the inverse problem. It would appear the BEM would be the method of choice for noisy data and when the heart is deep inside the torso, but MFS otherwise.

Conflicts of Interest: None Declared.

\section{References}

[1] Pullan, A. J., Cheng, L. K., Nash, M. P., Ghodrati, A., MacLeod, R., and Brooks, D. H. The inverse problem of electrocardiography In Macfarlane, P. W., van Oosterom, A., Pahlm, O., Kligfield, P., Janse, M., and Camm, J., editors, Comprehensive Electrocardiology, chapter 9, pages 299-344 Springer London, 2015/04/28/06:43:47 2010.

[2] Hansen, P. C. Analysis of discrete ill-posed problems by means of the L-curve SIAM Review, 34(4):561-580, 1992.

[3] Colli-Franzone, P., Guerri, L., Taccardi, B., and Viganotti, C. A numerical procedure to inversely compute epicardial potentials from body surface maps applied to a normal human subject In Computers in Cardiology, pages 187-190 IEEE Computer Society Press, 1981.

[4] Johnston, P. R. and Gulrajani, R. M. A new method for regularisation parameter determination in the inverse problem of electrocardiography IEEE Transactions on Biomedical Engineering, 44(1):19-39, 1997. 
[5] Wahba, G. Practical approximate solutions to linear operator equations when the data are noisy SIAM Journal on Numerical Analysis, 14:651-667, 1977.

[6] Lukas, M. A. Robust generalized cross-validation for choosing the regularization parameter Inverse Problems, 22(5):1883-1092, 2006.

[7] Barnes, J. P. and Johnston, P. R. Application of robust generalised cross-validation to the inverse problem of electrocardiology Computers in Biology and Medicine, 69:213-225, 2016.

[8] Bauer, F. and Lukas, M. A. Comparing parameter choice methods for regularization of ill-posed problems Mathematics and Computers in Simulation, 81(9):1795-1841, 2011/05/30/05:57:23 2011.

[9] Oster, H. S. and Rudy, Y. The use of temporal information in the regularisation of the inverse problem of electrocardiology IEEE Trans Biomed Engng, 39(1):65-75, January 1992.

[10] Huiskamp, G. and Greensite, F. A new method for myocardial activation imaging IEEE Transactions on Biomedical Engineering, 44(6):433-446, June 1997.

[11] Greensite, F. and Huiskamp, G. An improved method for estimating epicardial potentials from the body surface IEEE Transactions on Biomedical Engineering, 45(1):98-104, January 1998.

[12] Brooks, D. H., Ahmad, G. F., MacLeod, R. S., and Maratos, G. M. Inverse electrocardiography by simultaneous imposition of multiple constraints IEEE Transactions on Biomedical Engineering, 46(1):3-18, January 1999.

[13] Cheng, L. K., Bodley, J. M., and Pullan, A. J. Effects of experimental and modelling errors on electrocardiographic inverse formulations IEEE Transactions on Biomedical Engineering, 50(1):23-32, 2003.

[14] Gavgani, A. M. and Dogrusoz, Y. S. Use of genetic algorithm for selection of regularization parameters in multiple constraint inverse ECG problem In 2011 Annual International 
Conference of the IEEE Engineering in Medicine and Biology Society,EMBC, pages 985988, 2011.

[15] Gavgani, A. M. and Dogrusoz, Y. S. Noise reduction using anisotropic diffusion filter in inverse electrocardiology In 2012 Annual International Conference of the IEEE Engineering in Medicine and Biology Society (EMBC), pages 5919-5922, 2012.

[16] Dogrusoz, Y. S. and Gavgani, A. M. Genetic algorithm-based regularization parameter estimation for the inverse electrocardiography problem using multiple constraints Medical E Biological Engineering \& Computing, 51(4):367-375, 2013/05/03/04:23:36 2013.

[17] Wang, D., Kirby, R. M., and Johnson, C. R. Resolution strategies for the finite-elementbased solution of the ECG inverse problem IEEE Transactions on Biomedical Engineering, $57(2): 220-237,2010$.

[18] Wang, D., Kirby, R. M., MacLeod, R. S., and Johnson, C. R. Inverse electrocardiographic source localization of ischemia: An optimization framework and finite element solution Journal of Computational Physics, 250:403-424, 2013.

[19] Schulze, W. H. W., Wang, B., Potyagaylo, D., Dössel, O., and Long, M. Use of a tolerance operator in wavefront-based ecg imaging of transmembrane voltages In World Congress on Medical Physics and Biomedical Engineering May 26-31, 2012, Beijing, China, pages 422-425 Springer Berlin Heidelberg, 2013/03/05/09:50:18 2013.

[20] Wang, L. Computational reduction for noninvasive transmural electrophysiological imaging Computers in Biology and Medicine, 43(3):184-199, 2015/11/03/06:59:07 2013.

[21] Zemzemi, N. A steklov-poincaréapproach to solve the inverse problem in electrocardiography In Computing in Cardiology 2013, pages 703-706, 2013.

[22] Bouyssier, J., Zemzemi, N., and Henry, J. Inverse problem in electrocardiography via the factorization method of boundary value problems In 2015 IEEE 12th International Symposium on Biomedical Imaging (ISBI), pages 743-746, 2015. 
[23] Corrado, C., Gerbeau, J.-F., and Moireau, P. Identification of weakly coupled multiphysics problems. Application to the inverse problem of electrocardiography Journal of Computational Physics, 283:271-298, 2018/08/24/00:28:56 2015.

[24] Wang, Y. and Rudy, Y. Application of the method of fundamental solutions to potentialbased inverse electrocardiography Annals of Biomedical Engineering, 34(8):1272-1288, 2015/03/31/01:59:34 2006.

[25] Mathon, R. and Johnston, R. L. The approximate solution of elliptic boundary-value problems by fundamental solutions SIAM Journal on Numerical Analysis, 14(4):638-650, 2016/11/17/19:54:23 1977.

[26] Wei, T., Hon, Y. C., and Ling, L. Method of fundamental solutions with regularization techniques for cauchy problems of elliptic operators Engineering Analysis with Boundary Elements, 31(4):373-385, 2015/05/06/03:11:59 2007.

[27] Karageorghis, A., Lesnic, D., and Marin, L. A survey of applications of the MFS to inverse problems Inverse Problems in Science and Engineering, 19(3):309-336, 2015/05/11/22:26:05 2011.

[28] Chamorro-Servent, J., Bear, L., Duchateau, J., Dallet, C., Coudière, Y., and Dubois, R. Adaptive placement of the pseudo-boundaries improves the conditioning of the inverse problem In Computing in Cardiology, volume 43, pages 294-297, 2016.

[29] Chamorro-Servent, J., Dubois, R., and Coudière, Y. Exploring possible choices of the Tikhonov regularization parameter for the method of fundamental solutions in electrocardiography In Computing in Cardiology, volume 44, 2017.

[30] Chamorro-Servent, J., Dubois, R., Potse, M., and Coudière, Y. Improving the spatial solution of electrocardiographic imaging: A new regularization parameter choice technique for the Tikhonov method In International Conference on Functional Imaging and Modeling of the Heart, pages 289-300 Springer, Cham, 2018/05/28/06:48:56 2017.

[31] Gulrajani, R. M. Bioelectricity and Biomagnetism John Wiley and Sons, New York, 1998. 
[32] MacLeod, R. and Buist, M. The forward problem of electrocardiography In Macfarlane, P. W., Oosterom, A. v., Pahlm, O., Kligfield, P., Janse, M., and Camm, J., editors, Comprehensive Electrocardiology, chapter 8, pages 247-298 Springer London, 2015/04/28/07:03:18 2010.

[33] Claydon III, F. J., Pilkington, T. C., Tang, A. S. L., Morrow, M. N., and Ideker, R. E. A volume conductor model of the thorax for the study of defibrillation fields IEEE Transactions on Biomedical Engineering, 35(11):981-992, 1988.

[34] Tikhonov, A. N. and Arsenin, V. Y. Solutions of Ill-Posed Problems V. H. Winston and Sons, Washington, 1977.

[35] Aster, R. C., Borchers, B., and Thurber, C. H. Parameter Estimation and Inverse Problems Elsevier Academic Press, Burlington, 2005.

[36] Hansen, P. C. Rank-Deficient and Discrete Ill-Posed Problems SIAM, Philadelphia, 1998.

[37] Ni, Q., MacLeod, R. S., and Lux, R. L. Three-dimensional activation mapping in ventricular muscle: Interpolation and approximation of activation times Annals of Biomedical Engineering, 27:617-626, 1999.

[38] Horacek, B. M. Numerical model of an inhomogeneous human torso Advances in Cardiology, 10:51-57, 1974.

[39] Lorange, M. and Gulrajani, R. M. A computer heart model incorporating anisotropic propagation: I. Model construction and simulation of normal activation Journal of Electrocardiology, 26(4):245-261, 1993. 
Table Captions

Table 1: The number of times an optimal regularisation parameter determination method gave the minimum relative error at a given noise level for the normal heart beat. At each noise level, the methods are compared over all heart offsets and all time instants throughout the QRS complex.

Table 2: The number of times an optimal regularisation parameter determination method gave the minimum relative error at a given noise level for the ectopic beat. Comparisons are the same as those in Table 1.

Table 3: The number of times an optimal regularisation parameter determination method gave the minimum relative error at a given time instant throughout the normal heartbeat. At each time instant, the methods are compared over all heart offsets and all noise levels.

Table 4: The number of times an optimal regularisation parameter determination method gave the minimum relative error at a given time instant throughout the ectopic beat. Comparisons are the same as those in Table 3.

Table 5: The number of times an optimal regularisation parameter determination method gave the minimum relative error for the indicated heart offsets for the normal heartbeat. For each offset position, the methods are compared over all noise levels and all time instants. An offset of 0 indicates that the heart was in the correct position and, for example, an offset indicated by $+x$ means that the heart was offset in the positive $x$ direction by $10 \mathrm{~mm}$.

Table 6: The number of times an optimal regularisation parameter determination method gave the minimum relative error for the indicated heart offsets for the ectopic beat. Comparisons and notation are the same as those in Table 5.

Table 7: Number of times the respective regularisation parameter determination method yielded the lowest relative error across all 280 simulations for each of the normal and ectopic beats for both the BEM and MFS approaches.

\section{Figure Captions}

Figure 1: The heart-torso model considered in this study, showing the heart in the "correct" position. The two views are from the front (a) and from the left side (b). Here the torso consisted of 610 nodes and 1220 triangular elements and the heart had 114 nodes and 224 
elements. Overall, the torso was $550 \mathrm{~mm}$ high, $326 \mathrm{~mm}$ wide below the arms and $223 \mathrm{~mm}$ from front to back. The heart was $132 \mathrm{~mm}$ from base to apex and approximately $90 \mathrm{~mm}$ in diameter at the widest point.

Figure 2: Front (a) and back (b) views of the torso mesh with the dots indicating the positions of the 168 electrodes in the mapping system.

Figure 3: Node positions for the MFS simulations. White spheres indicate extended torso nodes (40mm in the direction of the outward pointing normal to the surface) and blue spheres indicate shifted heart nodes $(10 \mathrm{~mm}$ in the direction of the inward pointing normal to the surface).

Figure 4: Input epicardial potential distributions from a normal heartbeat used in the simulation study. The left column is for the 610 node heart and the right hand column is from the subsampled 114 node heart. The first row is at $205 \mathrm{~ms}$ into the heartbeat and the bottom row is $235 \mathrm{~ms}$ into the beat. Positive potentials are indicated by the red shading and solid contour lines and negative potentials are indicated by the blue shading and dashed contour lines. The maximum of the potential is indicated by the "+" sign and the minimum potential is indicated by the "-" sign. Maximum and minimum potentials, contour intervals and RMS values are indicated on each individual frame.

Figure 5: Input epicardial potential distributions from the ectopic beat used in the simulation study. The left column is for the 610 node heart and the right hand column is from the subsampled 114 node heart. The first row is at $130 \mathrm{~ms}$ into the heartbeat and the bottom row is $160 \mathrm{~ms}$ into the beat. The layout of the figure is the same as in Figure 4.

Figure 6: Epicardial potential distributions obtained from the indicated regularisation parameter determination method $205 \mathrm{~ms}$ into the normal heartbeat with $1 \%$ RMS noise added to the body surface potential distribution and the heart offset $10 \mathrm{~mm}$ in the positive $x$ direction (to the left). Results are presented for (a) the Optimal regularisation parameter (as defined in Section 2.5), and regularisation parameters determined by (b) GCV, (c) CRESO, (d) L-Curve, (e) Zero-Crossing and (f) RGCV. The regularisation parameter used in each case is also indicated. The layout of the figure is the same as in Figure 4 with the addition of Relative Error and Correlation Coefficient values compared to the 114 node distribution in Figure 4(b).

Figure 7: Epicardial potential distributions obtained from the indicated regularisation param- 
eter determination method $235 \mathrm{~ms}$ into the normal heartbeat with $20 \%$ RMS noise added to the body surface potential distribution and the heart offset $10 \mathrm{~mm}$ in the positive $z$ direction (backwards). The regularisation parameter used in each case is also indicated. The layout of the figure is the same as in Figure 6 with the Relative Error and Correlation Coefficient values compared to the 114 node distribution in Figure 4(d).

Figure 8: Epicardial potential distributions obtained from the indicated regularisation parameter determination method 130ms into the ectopic beat with 10\% RMS noise added to the body surface potential distribution and the heart offset $10 \mathrm{~mm}$ in the negative $z$ direction (forwards). The regularisation parameter used in each case is also indicated. The layout of the figure is the same as in Figure 6 with the Relative Error and Correlation Coefficient values compared to the 114 node distribution in Figure 5(b).

Figure 9: Epicardial potential distributions obtained from the indicated regularisation parameter determination method 160ms into the ectopic beat with $1 \%$ RMS noise added to the body surface potential distribution and the heart offset $10 \mathrm{~mm}$ in the positive $x$ direction (to the left). The regularisation parameter used in each case is also indicated. The layout of the figure is the same as in Figure 6 with the Relative Error and Correlation Coefficient values compared to the 114 node distribution in Figure 5(d).

Figure 10: Means of Relative Errors over 20 realisations for each regularisation parameter determination method at the indicated times during the QRS complex in the normal heartbeat. The error bars indicate the standard deviations about the mean for these 20 realisations. Here, $5 \%$ RMS noise was added to the body surface potential distribution and the heart was offset $10 \mathrm{~mm}$ in the positive $z$ direction (towards the back).

Figure 11: Means of Relative Errors over 20 realisations for each regularisation parameter determination method at the indicated times during the QRS complex in the normal heartbeat. The error bars indicate the standard deviations about the mean for these 20 realisations. Here, $20 \%$ RMS noise was added to the body surface potential distribution and the heart was offset $10 \mathrm{~mm}$ in the positive $z$ direction (towards the back).

Figure 12: Means of Relative Errors over 20 realisations for each regularisation parameter determination method at the indicated times during the QRS complex in the ectopic beat. The error bars indicate the standard deviations about the mean for these 20 realisations. Here, $10 \%$ 
RMS noise was added to the body surface potential distribution and the heart was offset $10 \mathrm{~mm}$ in the negative $z$ direction (towards the front).

Figure 13: Means of Relative Errors over 20 realisations for each regularisation parameter determination method at the indicated times during the QRS complex in the ectopic beat. The error bars indicate the standard deviations about the mean for these 20 realisations. Here, $1 \%$ RMS noise was added to the body surface potential distribution with the heart in the correct position. 


\begin{tabular}{|c|c|c|c|c|c|}
\hline $\begin{array}{c}\text { Added RMS } \\
\text { Noise }(\%)\end{array}$ & GCV & L-Curve & CRESO & Zero-X & RGCV \\
\hline 1 & 2 & 8 & 9 & 4 & 33 \\
2 & 3 & 7 & 15 & 4 & 27 \\
5 & 4 & 6 & 8 & 4 & 34 \\
10 & 12 & 5 & 4 & 2 & 33 \\
20 & 17 & 5 & 14 & 1 & 19 \\
\hline
\end{tabular}

Table 1:

\begin{tabular}{|c|c|c|c|c|c|}
\hline $\begin{array}{c}\text { Added RMS } \\
\text { Noise }(\%)\end{array}$ & GCV & L-Curve & CRESO & Zero-X & RGCV \\
\hline 1 & 7 & 5 & 21 & 0 & 23 \\
2 & 6 & 4 & 13 & 0 & 33 \\
5 & 19 & 4 & 1 & 0 & 32 \\
10 & 35 & 1 & 2 & 0 & 18 \\
20 & 23 & 0 & 7 & 1 & 25 \\
\hline
\end{tabular}

Table 2:

\begin{tabular}{|c|c|c|c|c|c|}
\hline Time $(\mathrm{ms})$ & GCV & L-Curve & CRESO & Zero-X & RGCV \\
\hline 175 & 4 & 1 & 3 & 0 & 27 \\
185 & 2 & 0 & 4 & 0 & 29 \\
195 & 4 & 2 & 7 & 8 & 14 \\
205 & 6 & 8 & 8 & 0 & 13 \\
215 & 4 & 15 & 11 & 0 & 5 \\
225 & 9 & 0 & 3 & 1 & 22 \\
235 & 6 & 0 & 7 & 0 & 22 \\
245 & 1 & 5 & 7 & 6 & 16 \\
\hline
\end{tabular}

Table 3: 


\begin{tabular}{|c|c|c|c|c|c|}
\hline Time $(\mathrm{ms})$ & GCV & L-Curve & CRESO & Zero-X & RGCV \\
\hline 90 & 20 & 0 & 2 & 0 & 13 \\
100 & 11 & 0 & 4 & 0 & 20 \\
110 & 13 & 0 & 4 & 0 & 18 \\
120 & 11 & 0 & 8 & 0 & 16 \\
130 & 11 & 0 & 6 & 1 & 17 \\
140 & 5 & 14 & 8 & 0 & 8 \\
150 & 11 & 0 & 6 & 0 & 18 \\
160 & 8 & 0 & 6 & 0 & 21 \\
\hline
\end{tabular}

Table 4:

\begin{tabular}{|c|c|c|c|c|c|}
\hline Offset & GCV & L-Curve & CRESO & Zero-X & RGCV \\
\hline 0 & 6 & 1 & 10 & 0 & 23 \\
$+x$ & 4 & 1 & 5 & 0 & 30 \\
$-x$ & 4 & 6 & 7 & 3 & 20 \\
$+y$ & 3 & 7 & 11 & 0 & 19 \\
$-y$ & 8 & 3 & 6 & 4 & 19 \\
$+z$ & 7 & 8 & 4 & 8 & 13 \\
$-z$ & 6 & 5 & 7 & 0 & 22 \\
\hline
\end{tabular}

Table 5: 


\begin{tabular}{|c|c|c|c|c|c|}
\hline Offset & GCV & L-Curve & CRESO & Zero-X & RGCV \\
\hline 0 & 16 & 1 & 10 & 0 & 13 \\
$+x$ & 17 & 0 & 11 & 0 & 12 \\
$-x$ & 9 & 3 & 0 & 0 & 28 \\
$+y$ & 11 & 3 & 4 & 1 & 21 \\
$-y$ & 11 & 3 & 8 & 0 & 18 \\
$+z$ & 18 & 4 & 6 & 0 & 12 \\
$-z$ & 8 & 0 & 5 & 0 & 27 \\
\hline
\end{tabular}

Table 6:

\begin{tabular}{|l|c|c|c|c|}
\hline & \multicolumn{2}{|c|}{ BEM } & \multicolumn{2}{c|}{ MFS } \\
\hline & Normal Beat & Ectopic Beat & Normal Beat & Ectopic Beat \\
\hline RGCV & 133 & 110 & 146 & 131 \\
CRESO & 26 & 27 & 50 & 44 \\
GCV & 10 & 11 & 38 & 90 \\
L-Curve & 56 & 85 & 31 & 14 \\
Zero-Crossing & 55 & 47 & 15 & 1 \\
\hline
\end{tabular}

Table 7: 


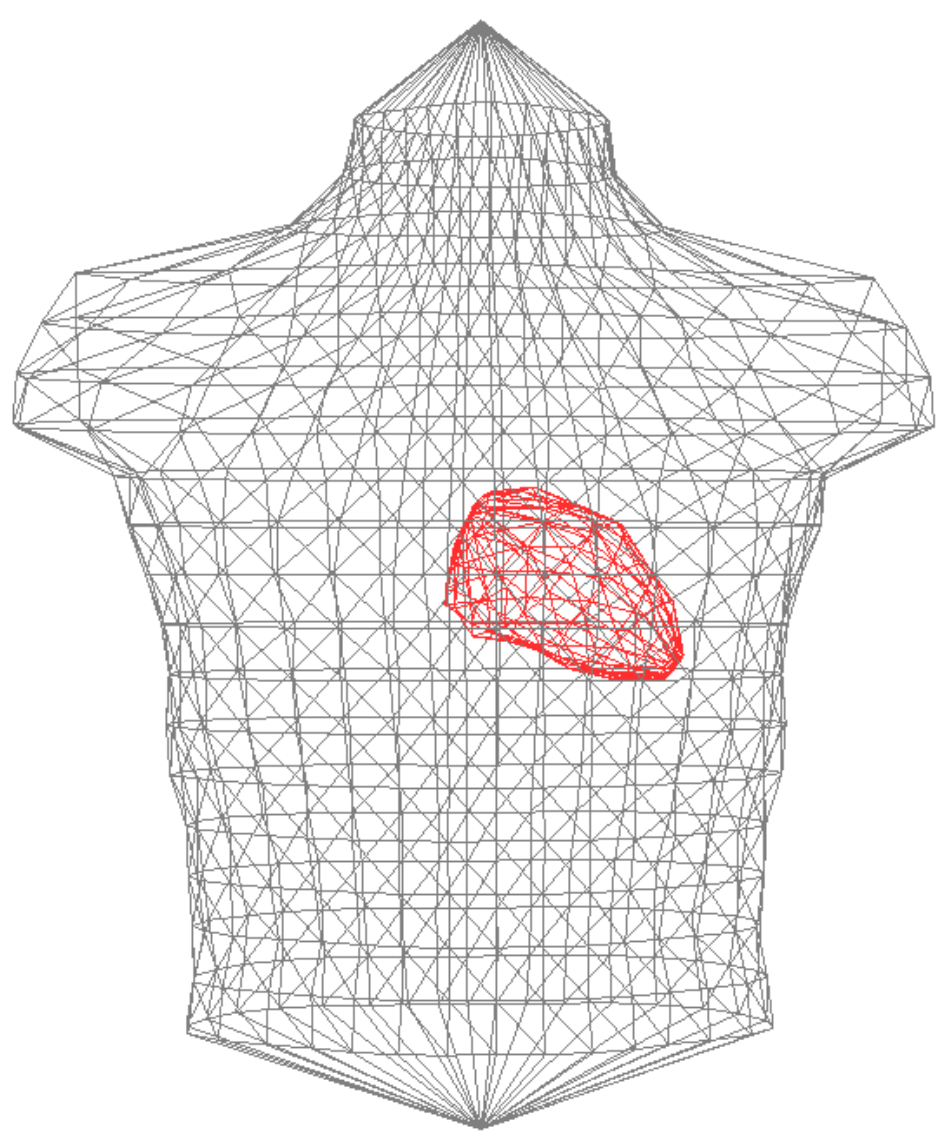

(a) Front View

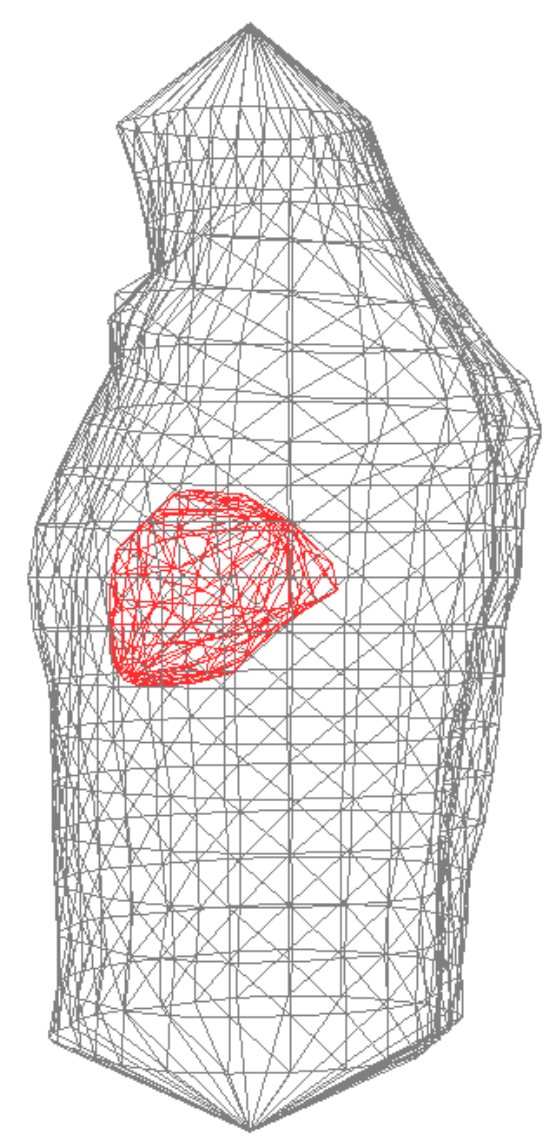

(b) Left View

Figure 1: 


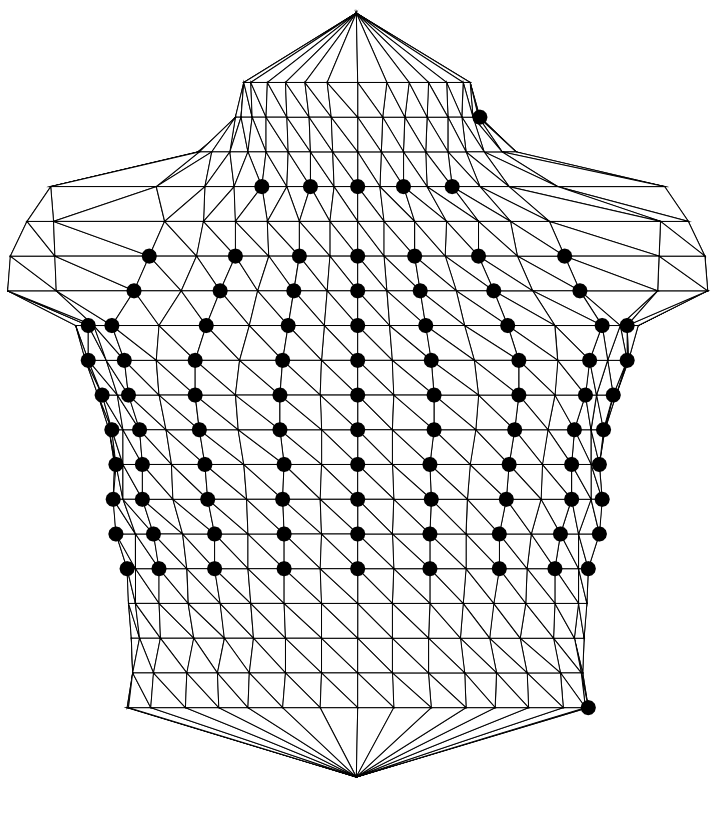

(a) Front

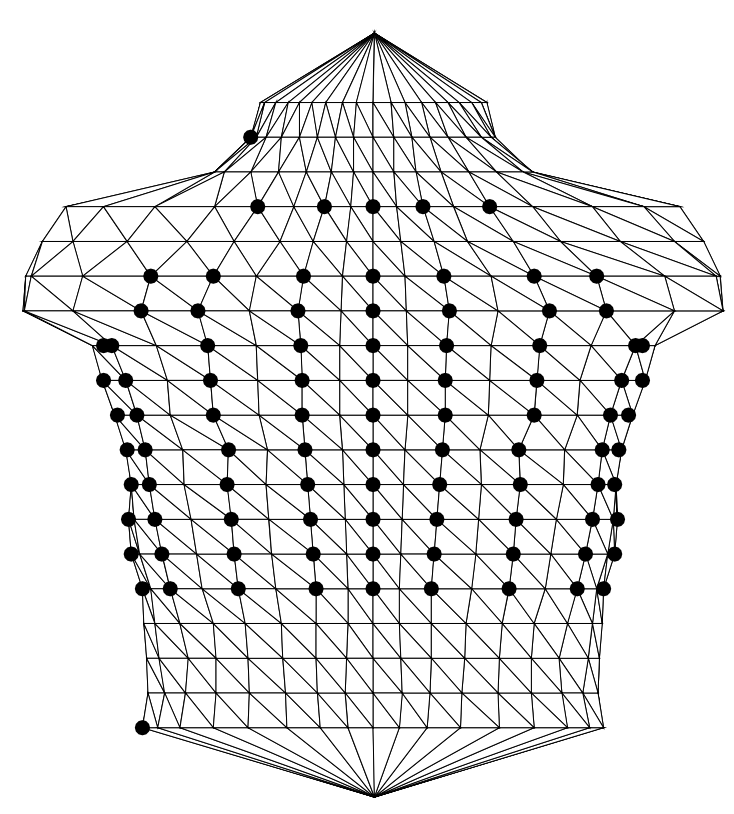

(b) Back

Figure 2: 


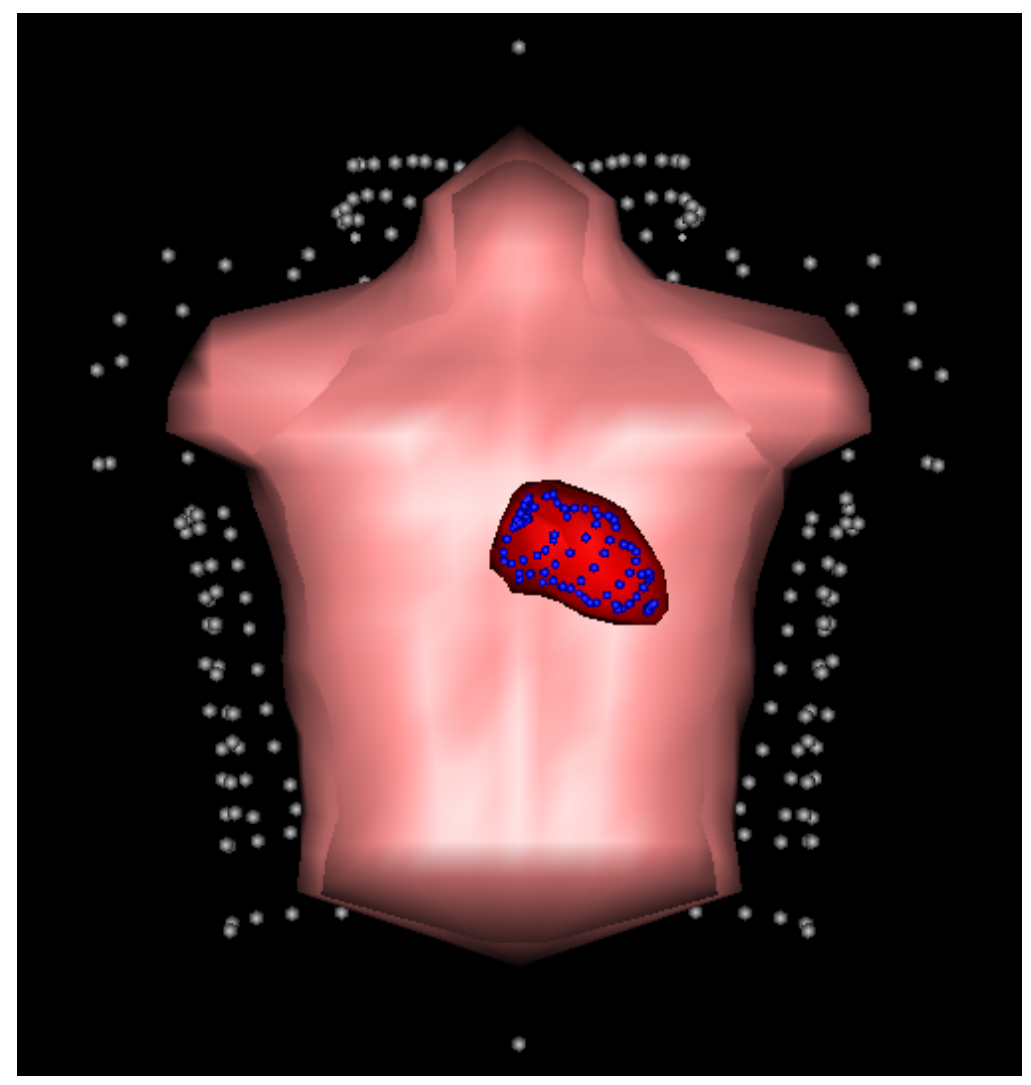

Figure 3: 


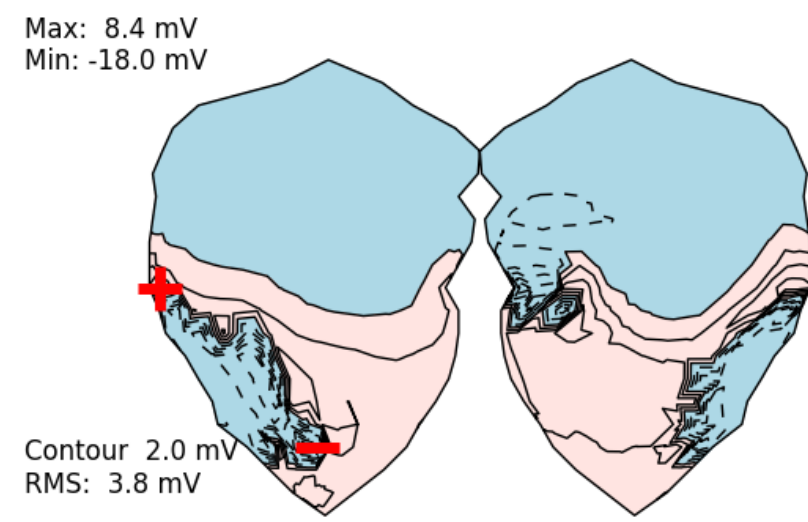

(a) time $=205 \mathrm{~ms}, 610$ node mesh

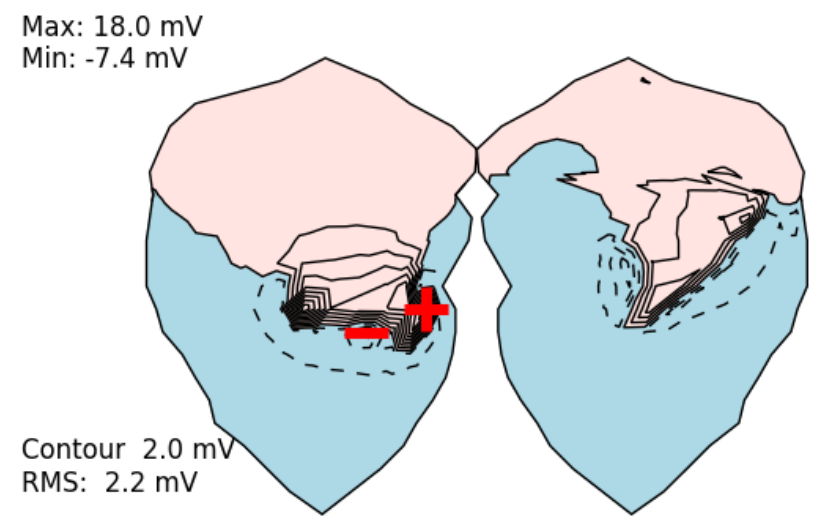

(c) time $=235 \mathrm{~ms}, 610$ node mesh

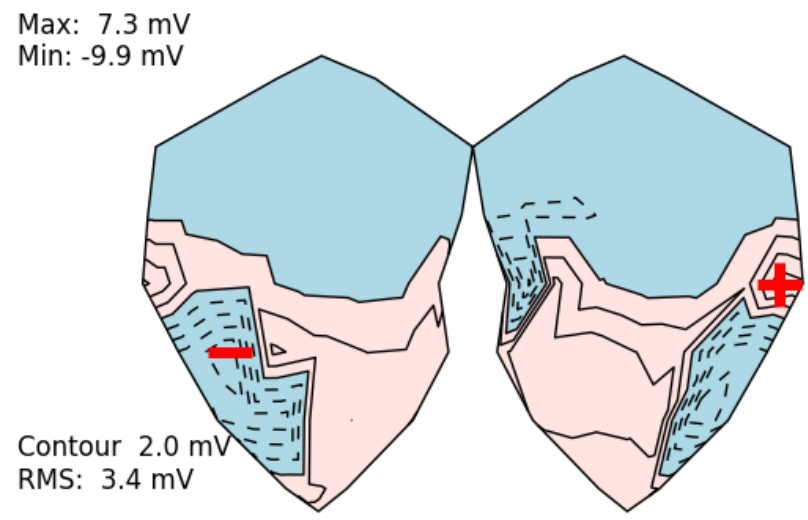

(b) time $=205 \mathrm{~ms}, 114$ node mesh

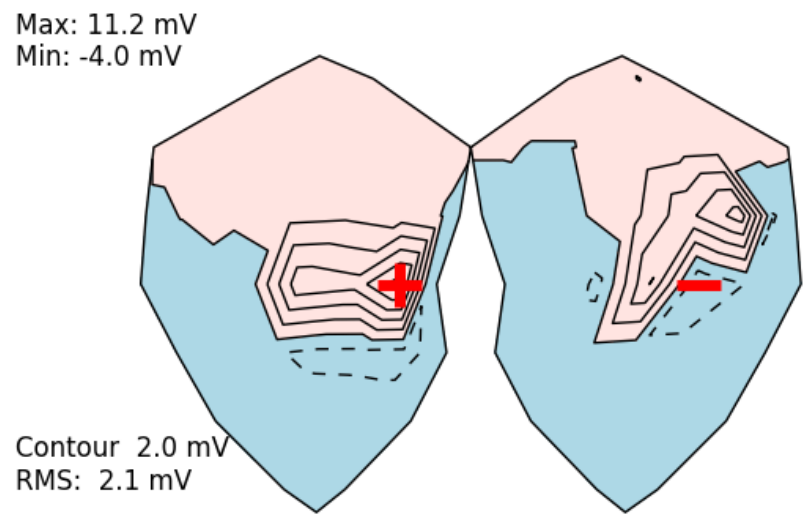

(d) time $=235 \mathrm{~ms}, 114$ node mesh

Figure 4: 


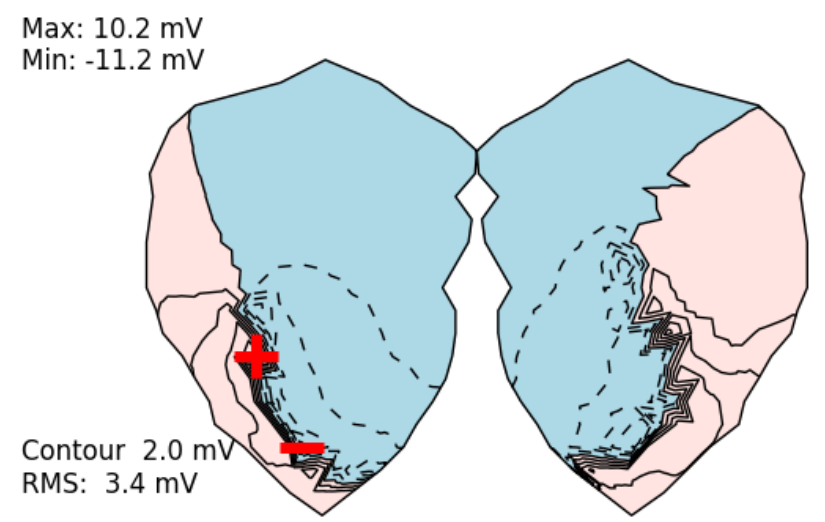

(a) time $=130 \mathrm{~ms}, 610$ node mesh

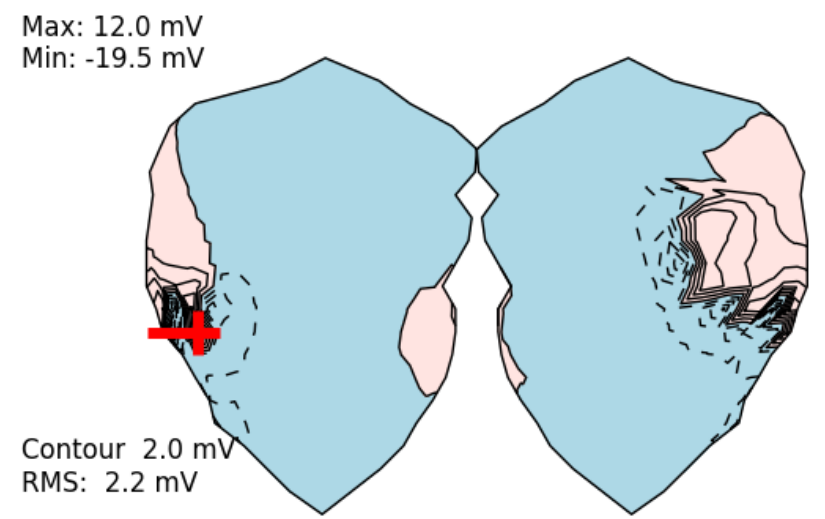

(c) time $=160 \mathrm{~ms}, 610$ node mesh

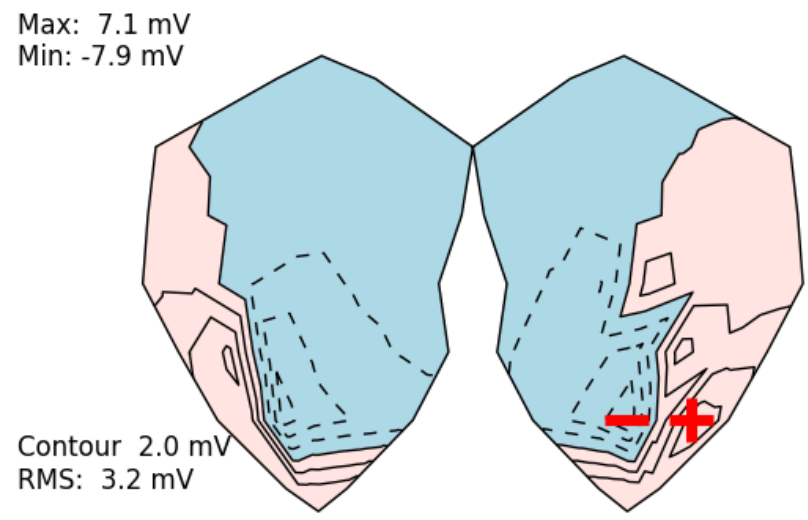

(b) time $=130 \mathrm{~ms}, 114$ node mesh

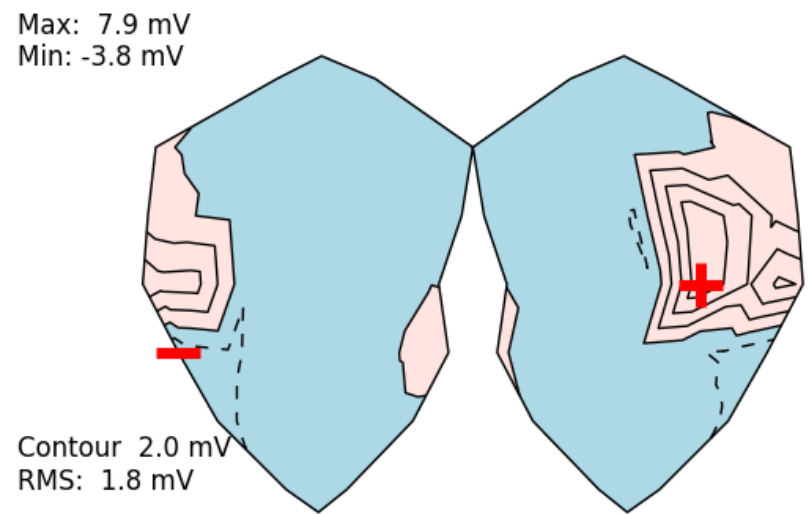

(d) time $=160 \mathrm{~ms}, 114$ node mesh

Figure 5: 


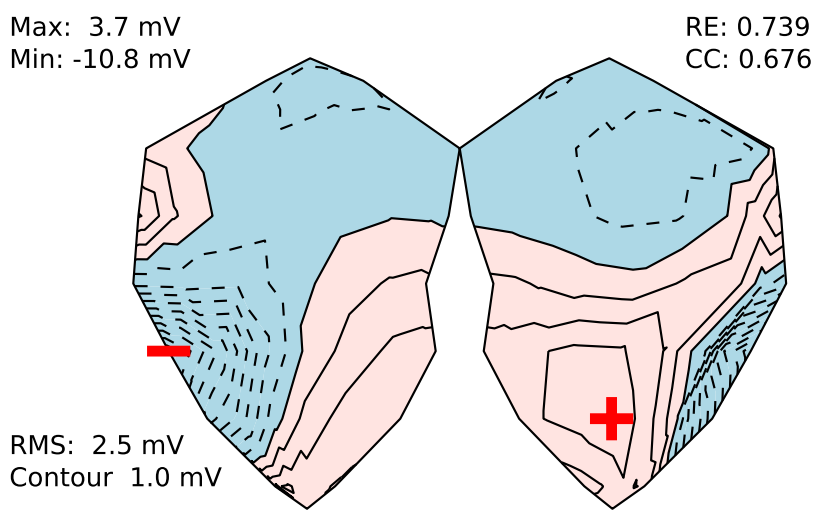

(a) Optimal $\left(\lambda=9.32 \times 10^{-6}\right)$

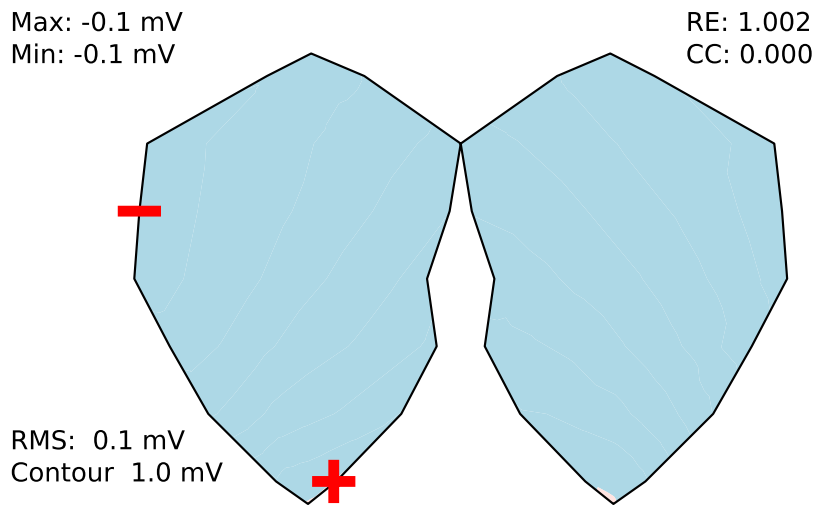

(c) $\operatorname{CRESO}(\lambda=2.27)$

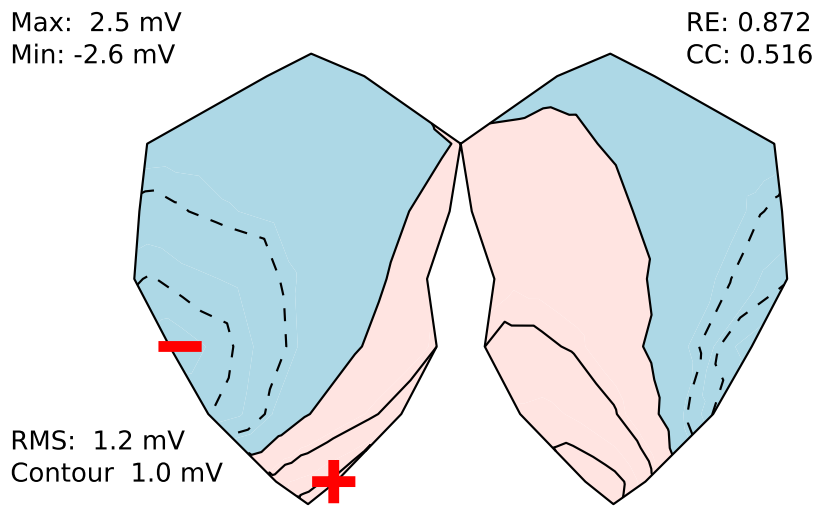

(e) Zero-X $\left(\lambda=3.49 \times 10^{-3}\right)$

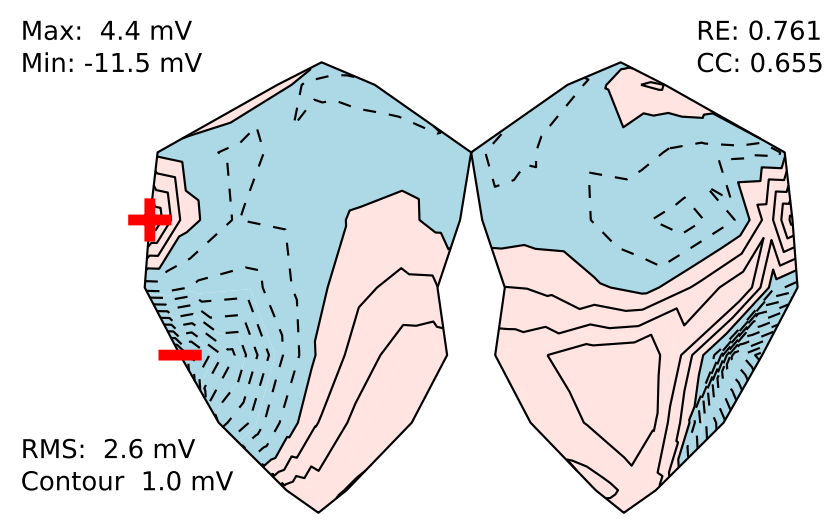

(b) $\operatorname{GCV}\left(\lambda=3.70 \times 10^{-6}\right)$
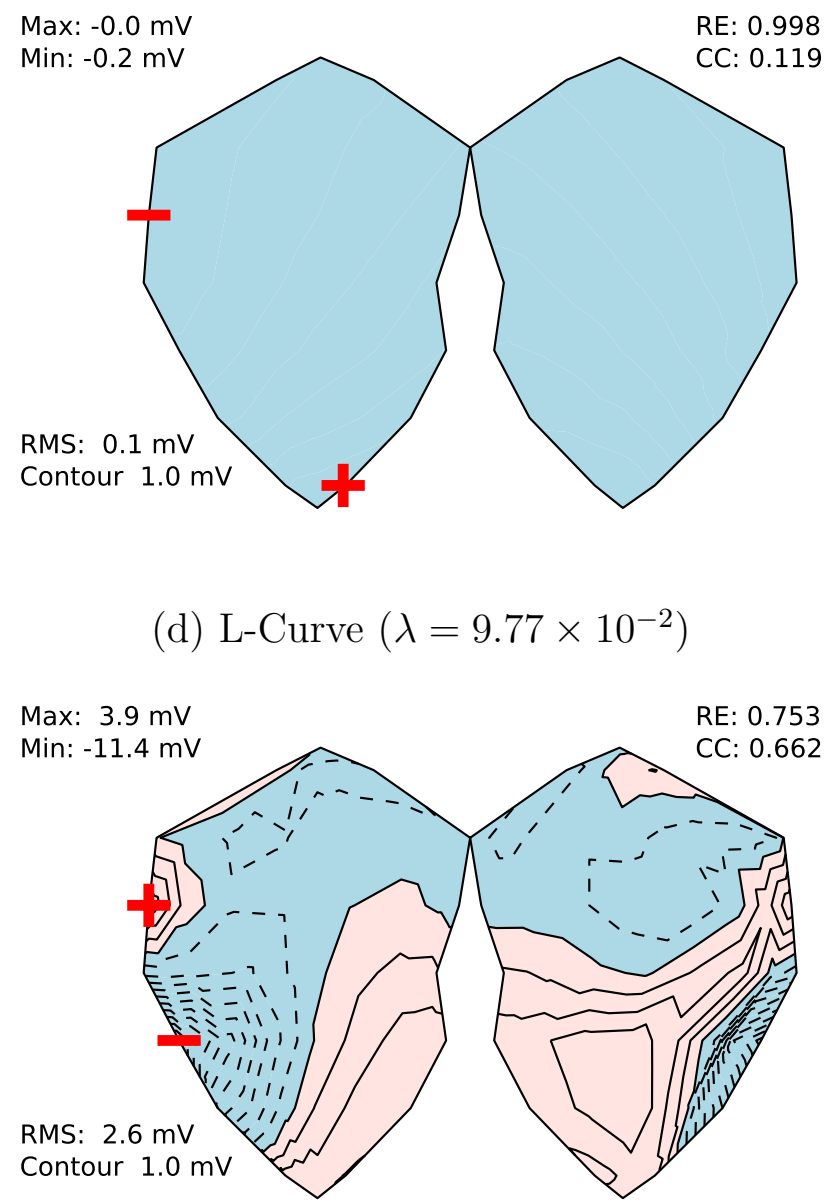

(f) $\operatorname{RGCV}\left(\lambda=5.35 \times 10^{-6}\right)$

Figure 6: 
Max: $2.2 \mathrm{mV}$

Min: $-2.2 \mathrm{mV}$

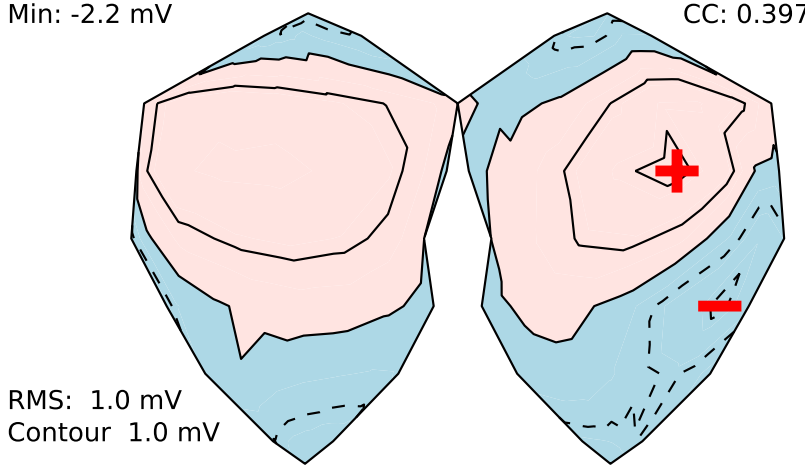

(a) Optimal $\left(\lambda=3.11 \times 10^{-5}\right)$

Max: $3.5 \mathrm{mV}$
Min: $-2.8 \mathrm{mV}$

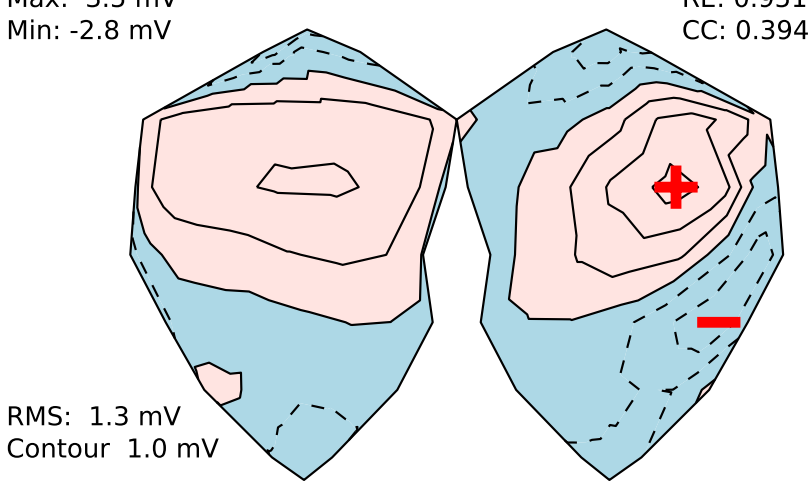

(c) $\operatorname{CRESO}\left(\lambda=1.35 \times 10^{-5}\right)$

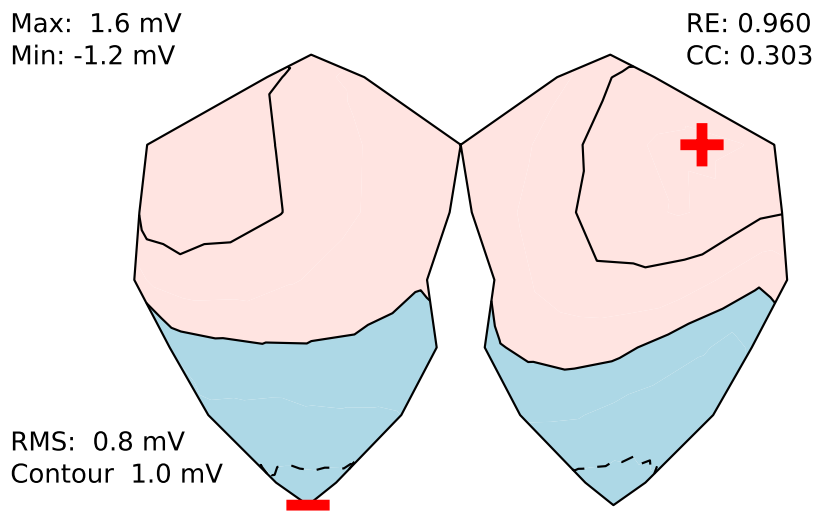

(e) Zero-X $\left(\lambda=1.50 \times 10^{-4}\right)$

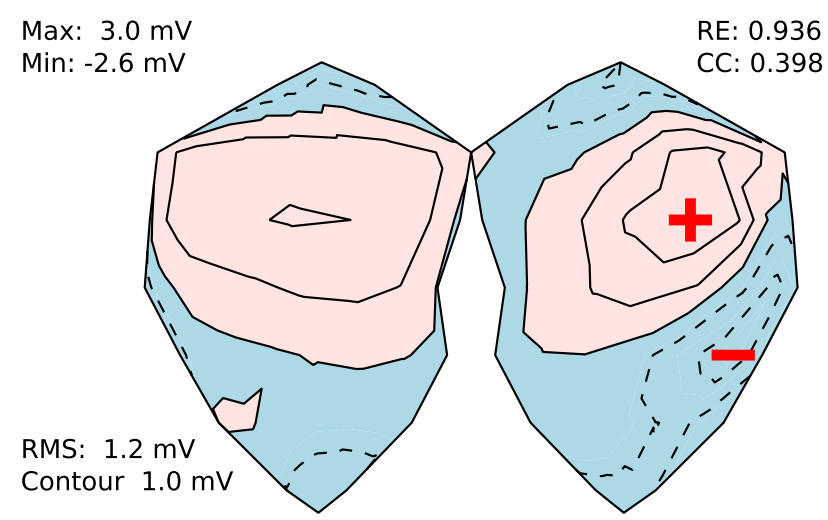

(b) $\operatorname{GCV}\left(\lambda=1.78 \times 10^{-5}\right)$

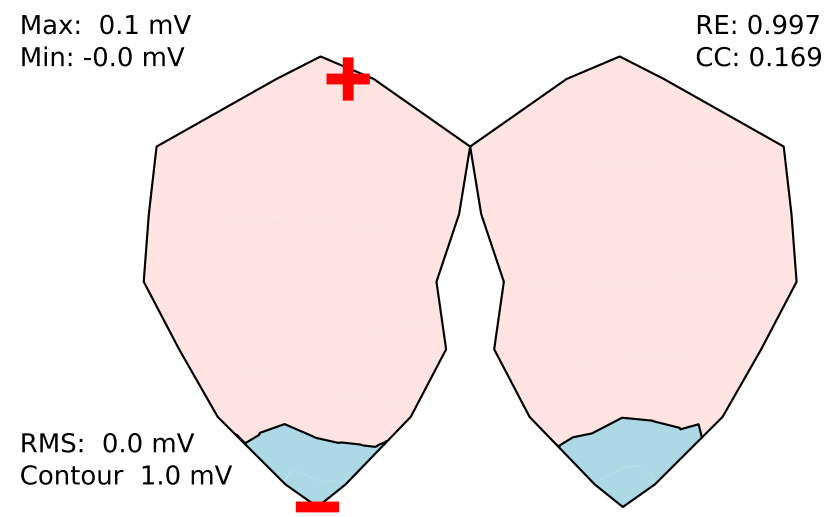

(d) L-Curve $\left(\lambda=1.18 \times 10^{-1}\right)$

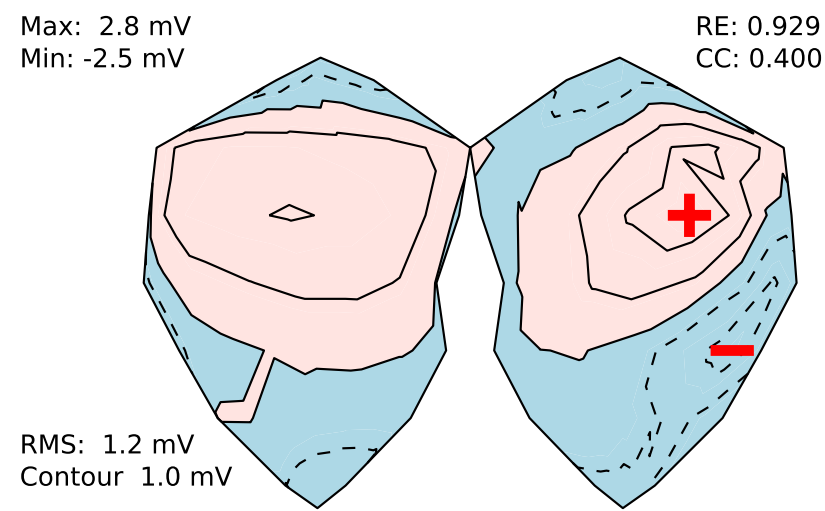

(f) $\operatorname{RGCV}\left(\lambda=2.15 \times 10^{-5}\right)$

Figure 7: 


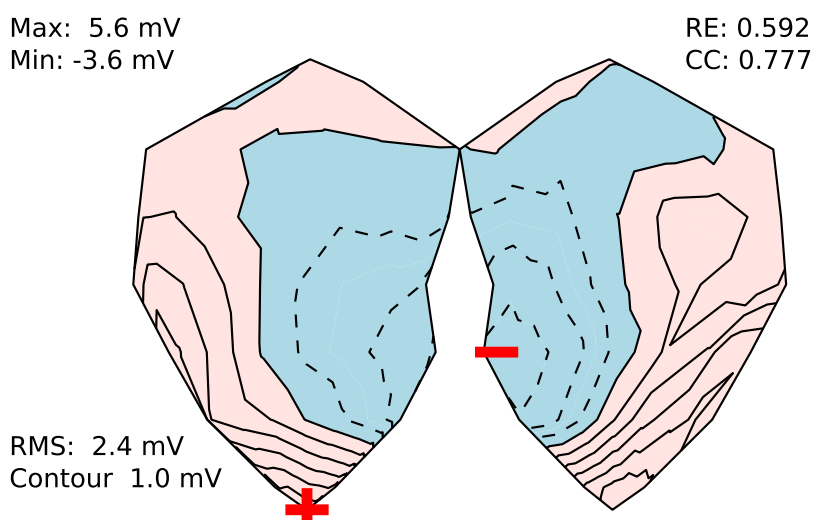

(a) Optimal $\left(\lambda=3.74 \times^{-5}\right)$

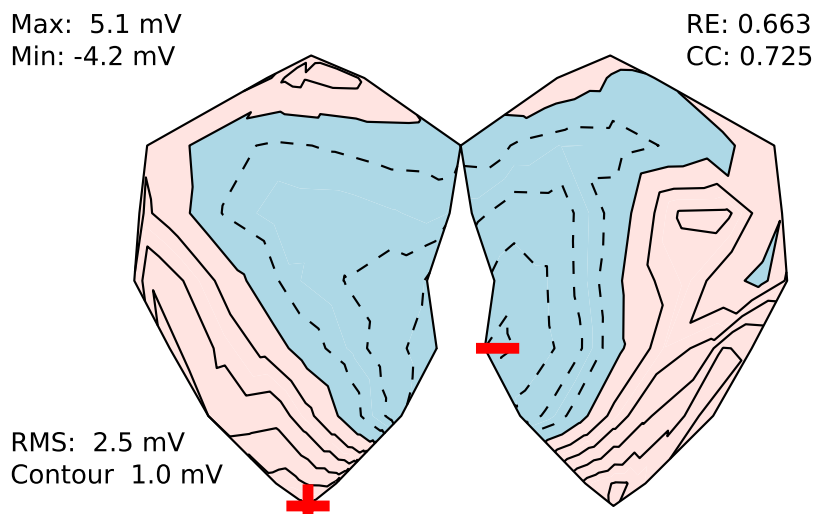

(c) $\operatorname{CRESO}\left(\lambda=1.12 \times 10^{-5}\right)$

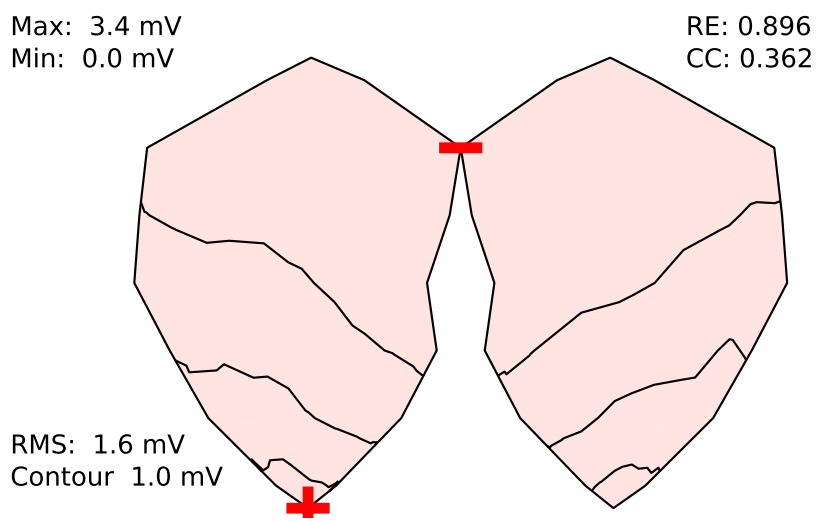

(e) Zero-X $\left(\lambda=1.40 \times 10^{-2}\right)$

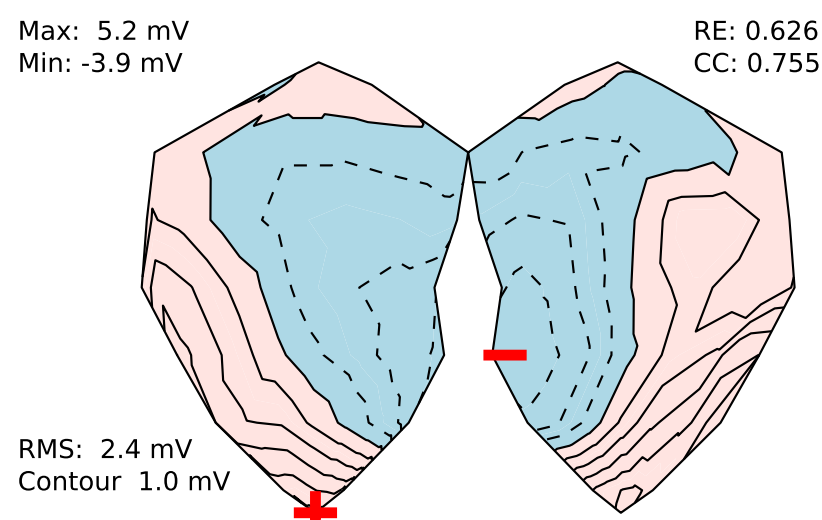

(b) $\operatorname{GCV}\left(\lambda=1.78 \times 10^{-5}\right)$
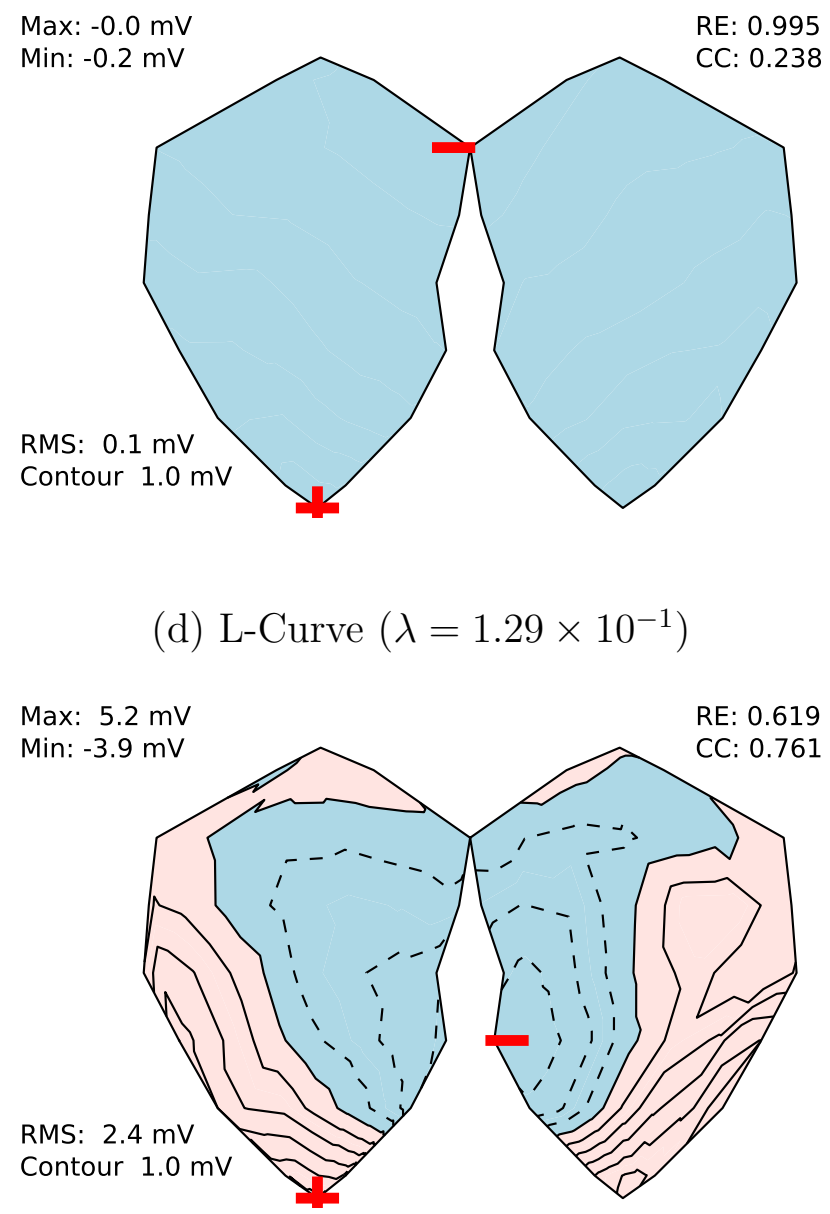

(f) $\operatorname{RGCV}\left(\lambda=1.95 \times 10^{-5}\right)$

Figure 8: 
Max: $4.7 \mathrm{mV}$

RMS: $1.5 \mathrm{mV}$

Contour $1.0 \mathrm{mV}$
Min: $-3.8 \mathrm{mV}$

RE: 0.730

CC: 0.667

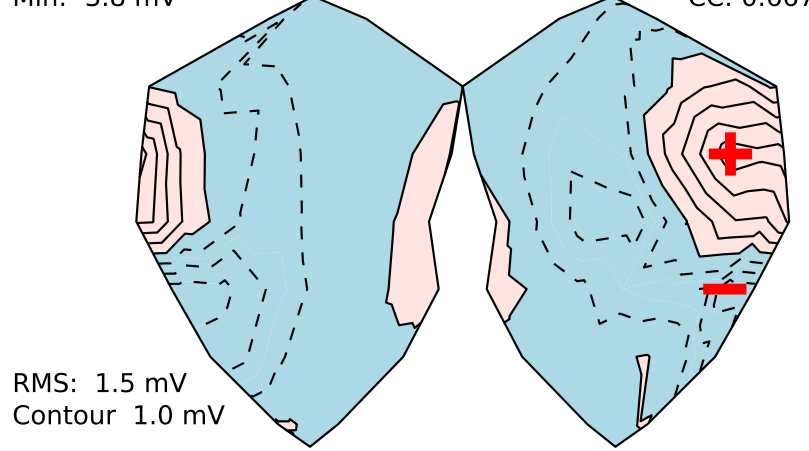

(a) Optimal $\left(\lambda=5.35 \times 10^{-6}\right)$

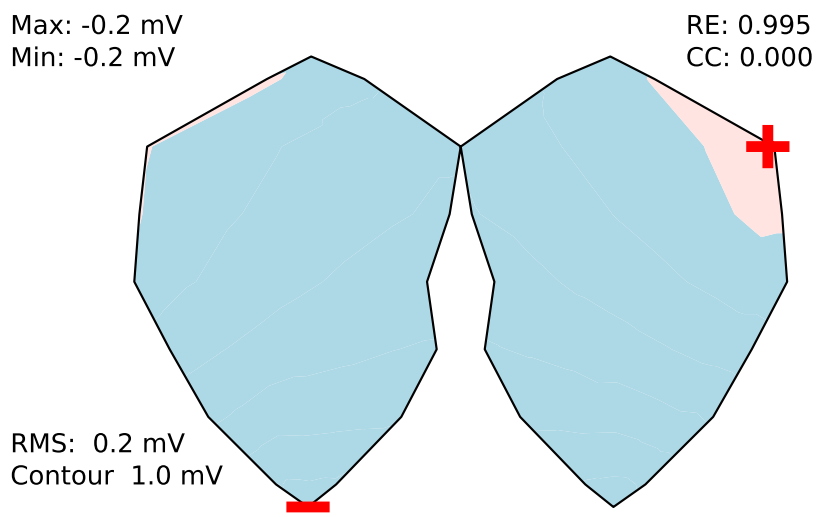

(c) $\operatorname{CRESO}(\lambda=1.43)$

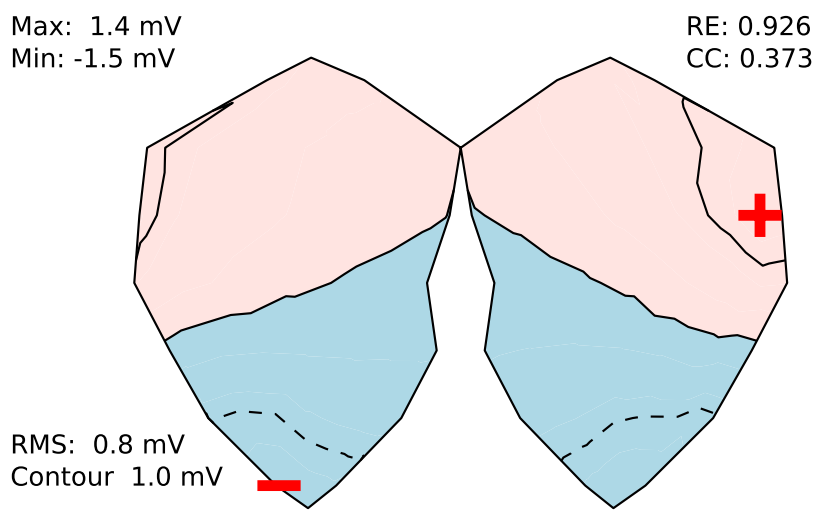

(e) Zero-X $\left(\lambda=3.59 \times 10^{-6}\right)$

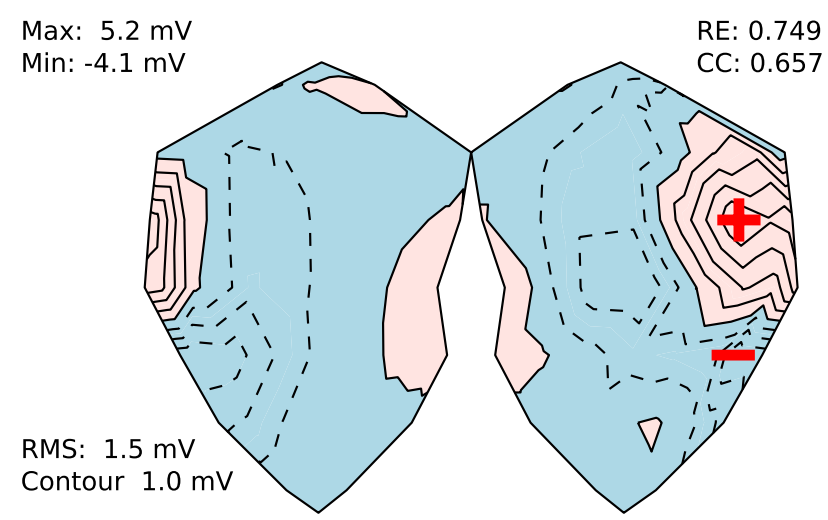

(b) $\operatorname{GCV}\left(\lambda=2.55 \times 10^{-6}\right)$

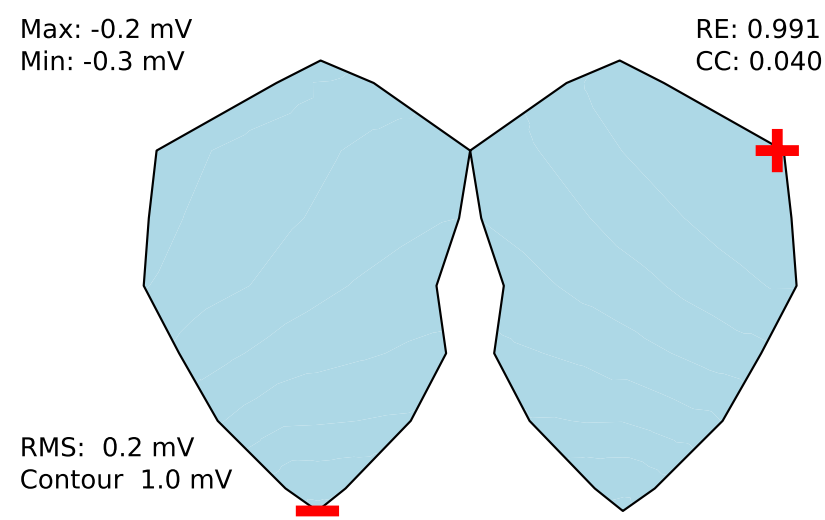

(d) L-Curve $\left(\lambda=8.91 \times 10^{-2}\right)$

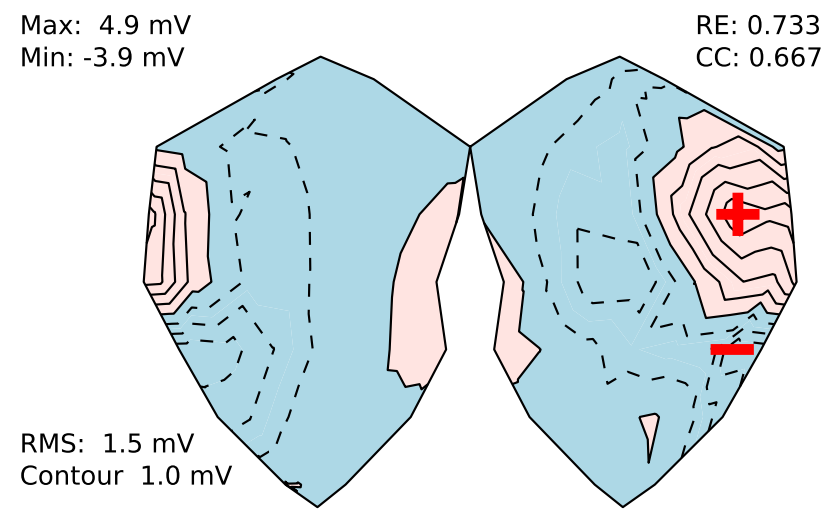

(f) $\operatorname{RGCV}\left(\lambda=4.06 \times 10^{-6}\right)$

Figure 9: 


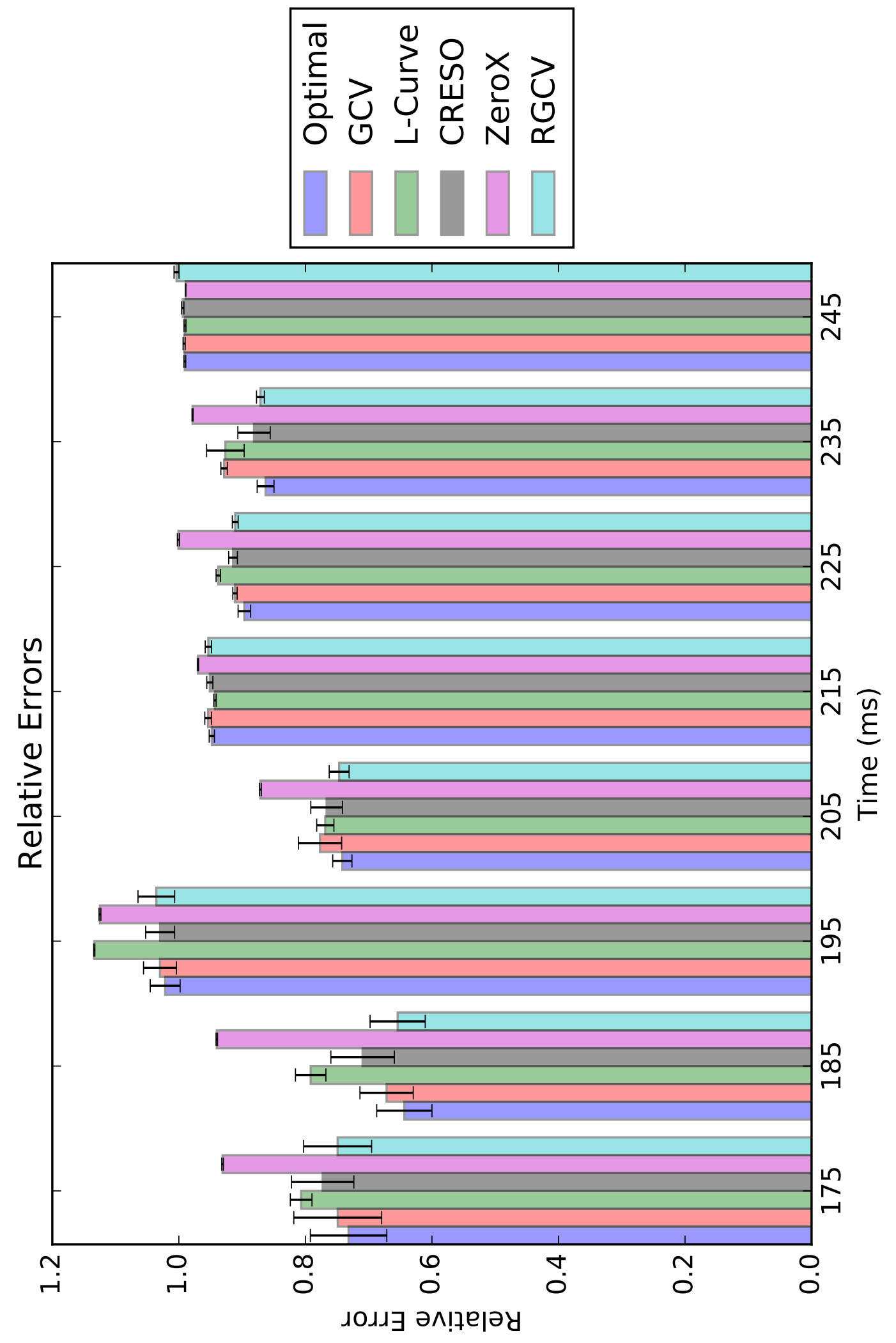

Figure 10: 


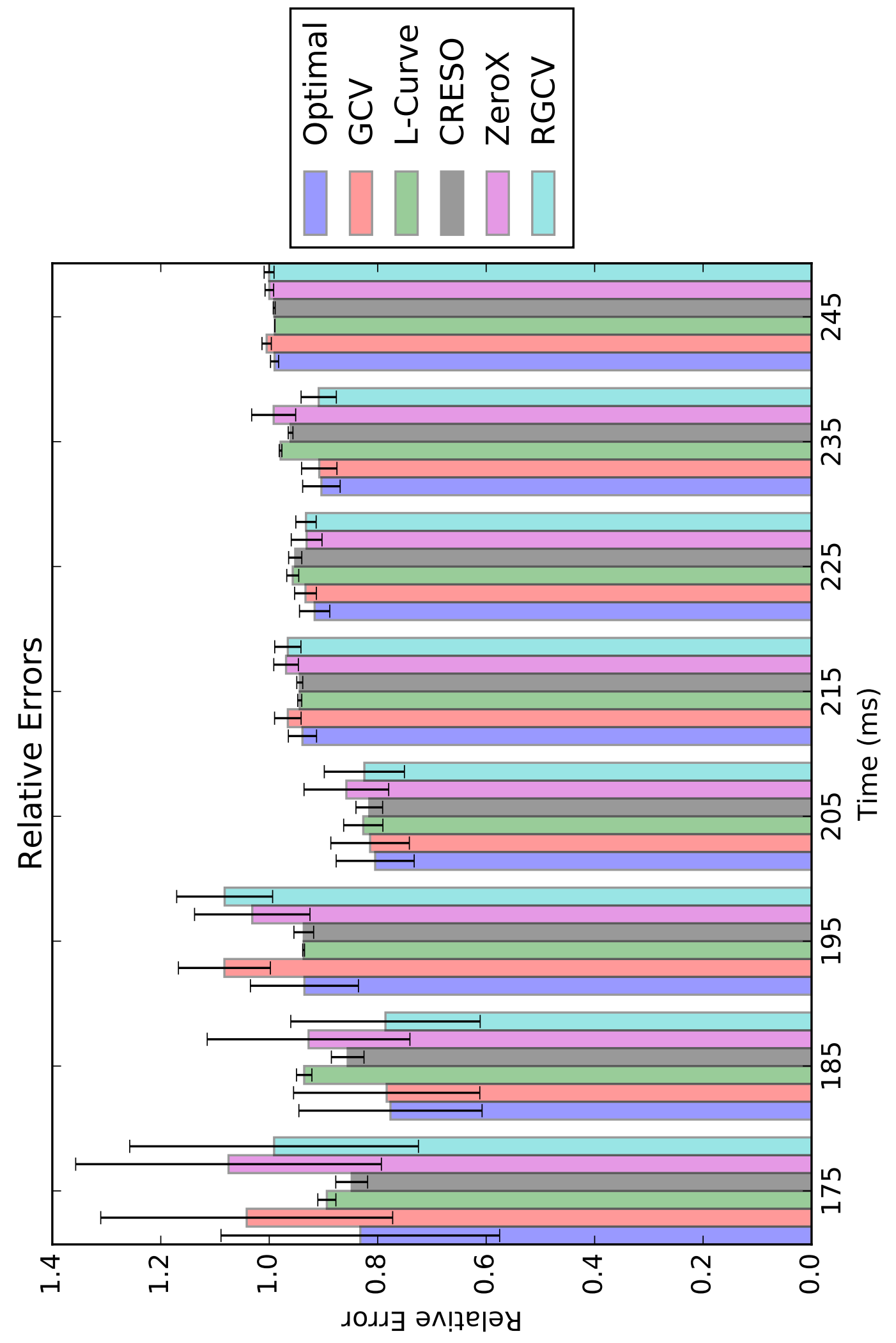

Figure 11: 


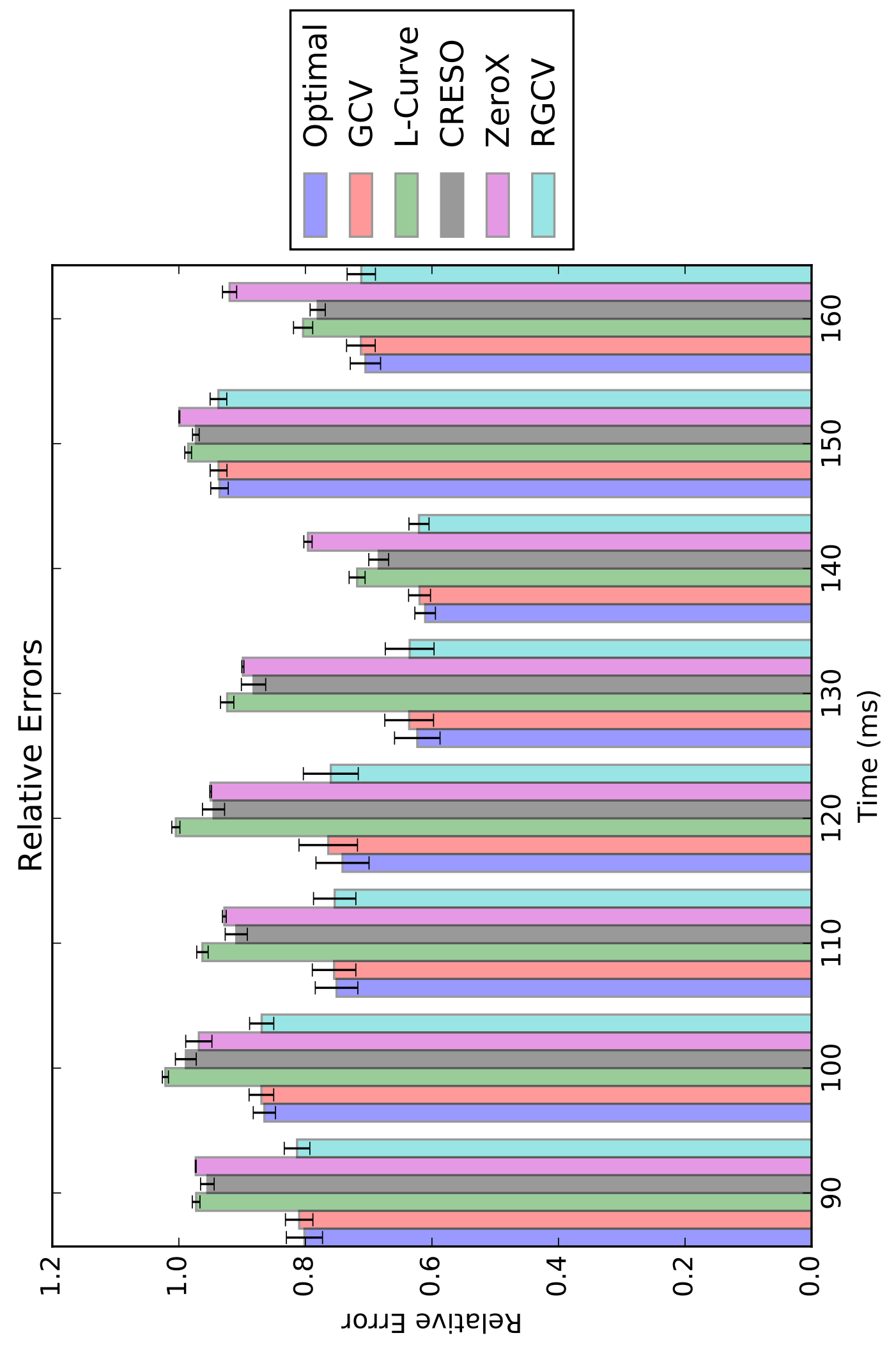

Figure 12: 


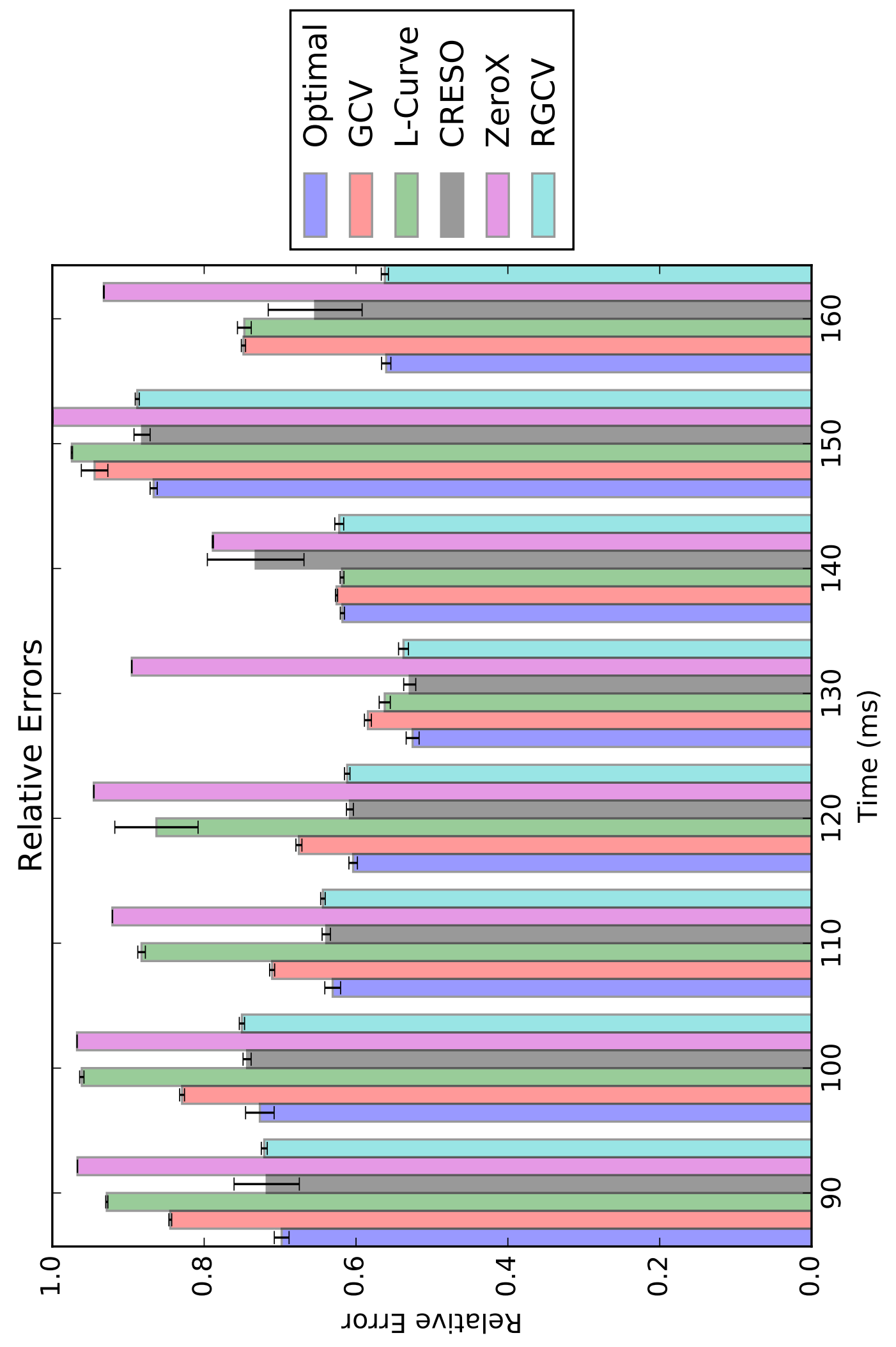

Figure 13: 